\title{
Partial interior regularity for sub-elliptic systems with Dini continuous coefficients in Carnot groups: the sub-quadratic controllable case
}

\author{
Dongni Liao ${ }^{1}$, Jialin Wang ${ }^{1 *}$, Qiang Yang ${ }^{1}$ and Shimin Wu ${ }^{2}$
}

\author{
${ }^{*}$ Correspondence: \\ jialinwang1025@hotmail.com \\ ${ }^{1}$ School of Mathematics and \\ Computer Science, Gannan Normal \\ University, Ganzhou, Jiangxi 341000, \\ P.R. China \\ Full list of author information is \\ available at the end of the article
}

\begin{abstract}
We consider nonlinear sub-elliptic systems with Dini continuous coefficients for the case $1<m<2$ in Carnot groups and prove a $C^{1}$-partial regularity result for weak solutions under the controllable growth conditions. Our method of proof for sub-elliptic systems is based on a generalization of the technique of $\mathcal{A}$-harmonic approximation. It is interesting to point out that our result is optimal in the sense that in the case of Hölder continuous coefficients we get directly the optimal Hölder exponent on its regular set.
\end{abstract}

MSC: $35 \mathrm{H} 20 ; 35 \mathrm{~B} 65$

Keywords: nonlinear sub-elliptic system; Dini continuous coefficients; sub-quadratic controllable growth; optimal partial regularity; Carnot groups

\section{Introduction and the main result}

In this paper, we are focused on the nonlinear sub-elliptic systems involving sub-quadratic $(1<m<2)$ controllable growth terms in divergence form in Carnot groups $G$,

$$
-\sum_{i=1}^{k} X_{i} A_{i}^{\alpha}(\xi, u, X u)=B^{\alpha}(\xi, u, X u), \quad \xi \in \Omega, \alpha=1, \ldots, N,
$$

where $\Omega$ is a bounded domain in $G, X=\left\{X_{1}, \ldots, X_{k}\right\}$ with $X_{i}(i=1, \ldots, k)$ to see (2.1) below, $u: \Omega \rightarrow \mathbb{R}^{N}, A_{i}^{\alpha}: \Omega \times \mathbb{R}^{N} \times \mathbb{R}^{k \times N} \rightarrow \mathbb{R}^{k \times N}$, and $B^{\alpha}: \Omega \times \mathbb{R}^{N} \times \mathbb{R}^{k \times N} \rightarrow \mathbb{R}^{N}$.

The aim is to weaken assumptions on coefficients $A_{i}^{\alpha}$ with Hölder continuity in the variables $(\xi, u)$ to the assumptions of Dini continuity, and to show a partial regularity result with optimal estimates for the modulus of continuity for the horizontal derivative $\mathrm{Xu}$; see $[1,2]$ for the case of sub-elliptic systems with Hölder continuous coefficients.

As is well known, even under reasonable assumptions on the coefficients $A_{i}^{\alpha}, B^{\alpha}$ in the systems, people cannot in general expect that weak solutions of nonlinear elliptic systems of equations will be classical (i.e., $C^{2}$-solutions) like elliptic scalar equations; see [3] by De Giorgi. Then the goal is to establish partial regularity of weak solutions for systems; see monographs [4-6] for more details. Moreover, Duzaar and Steffen in [7] introduced a

(c) The Author(s) 2017. This article is distributed under the terms of the Creative Commons Attribution 4.0 International License (http://creativecommons.org/licenses/by/4.0/), which permits unrestricted use, distribution, and reproduction in any medium, provided you give appropriate credit to the original author(s) and the source, provide a link to the Creative Commons license, and indicate if changes were made. 
new method called $\mathcal{A}$-harmonic approximation technique, which was then simplified by Duzaar and Grotowski in [8], to investigate elliptic systems with quadratic growth case. This technique avoids proving a reverse Hölder inequality. Later, many results have been obtained for more general coefficients $A_{i}^{\alpha}$ or $B^{\alpha}$ in the Euclidean setting; see [9-12] for Hölder continuous coefficients, and [13-15] for Dini continuous coefficients.

With respect to sub-elliptic systems in Carnot groups, there are some new difficulties and challenges which remain due to the lack of commutation and homogeneity of the horizontal vector fields. We list several results for sub-elliptic systems with Hölder continuous coefficients. Capogna and Garofalo [16], and Shores [17] considered the quadratic growth case, whereas Föglein [18], Wang and Niu [1], and Wang and Liao [2] treated non-quadratic cases. It is remarkable that Zheng and Feng [19] showed everywhere regularity for weak solutions of sub-elliptic $p$-harmonic systems while $p$ is closed to 2 in Carnot groups. We also refer readers to [20-25] for regularity of weak solutions and to [26-30] for other interesting topics such as variational principle, uniqueness, existence and nonexistence of solutions to sub-elliptic equations on Carnot groups.

Recently, Wang and Liao [31] considered sub-elliptic systems with Dini continuous coefficients under super-quadratic conditions $(m \geq 2)$. In this paper, we treat the sub-quadratic case $(1<m<2)$. It is worth mentioning that the key point is to establish a certain excessdecay estimate for the excess functional $\Phi$. In the case $m \geq 2$, this functional is given by

$$
\Phi\left(\xi_{0}, \rho, p_{0}\right)=f_{B_{\rho}\left(\xi_{0}\right)}\left[\left|X u-p_{0}\right|^{2}+\left|X u-p_{0}\right|^{m}\right] d \xi
$$

where we have used the notation $f_{B_{r}\left(\xi_{0}\right)} u(\xi) d \xi=\frac{1}{\left|B_{r}\left(\xi_{0}\right)\right|_{G}} \int_{B_{r}\left(\xi_{0}\right)} u(\xi) d \xi$. However, in the sub-quadratic case $1<m<2$, one should establish the excess-decay estimate for the following functional:

$$
\Phi\left(\xi_{0}, \rho, p_{0}\right)=f_{B_{\rho}\left(\xi_{0}\right)}\left|V(X u)-V\left(p_{0}\right)\right|^{2} d \xi
$$

where $V(A)=\left(1+|A|^{2}\right)^{\frac{4}{m-2}}$ for $A \in \mathbb{R}^{k \times N}$, which itself leads to the continuity of the horizontal gradient $X u$ of the weak solution via the integral characterization of continuity by Campanato. For $1<m<2$, we first have to generalize the approximation lemma directly (see Lemma 4 below), and the proof requires a Sobolev-Poincaré type inequality related to the functions $V$ (see Lemma 5 below).

In the sequel, let $\Omega \subset G$ be a bounded domain in Carnot groups $G$ with general step and consider weak solutions of the sub-elliptic systems (1.1), i.e.,

$$
\int_{\Omega} A_{i}^{\alpha}(\xi, u, X u) X_{i} \varphi^{\alpha} d \xi=\int_{\Omega} B^{\alpha}(\xi, u, X u) \varphi^{\alpha} d \xi, \quad \forall \varphi \in C_{0}^{\infty}\left(\Omega, \mathbb{R}^{N}\right)
$$

with sub-quadratic controllable structure conditions $(\mathrm{H} 1)-(\mathrm{H} 4)$, where:

$(\mathrm{H} 1): A_{i}^{\alpha}(\xi, u, p)$ is differentiable with respect to $p$, with bounded and continuous derivatives, that is, there exists a constant $C$ such that

$$
\left|A_{i, p_{\beta}^{\prime}}^{\alpha}(\xi, u, p)\right| \leq C\left(1+|p|^{2}\right)^{\frac{m-2}{2}}, \quad 1<m<2
$$

where we denote by $A_{i, p_{\beta}^{j}}^{\alpha}(\cdot)=\frac{\partial A_{i}^{\alpha} \cdot(\cdot)}{\partial p_{\beta}^{j}}$. Furthermore, (H1) implies that there exists a continuously nonnegative and bounded function $\omega(s, t):[0, \infty) \times[0, \infty) \rightarrow[0, \infty)$, where 
$\omega(s, 0)=0$ for all $s$, and $\omega(s, t)$ is monotonously nondecreasing in $s$ for fixed $t ; \omega(s, t)$ is concave and monotonously nondecreasing in $t$ for fixed $s$ such that

$$
\left|A_{i, p_{\beta}^{j}}^{\alpha}(\xi, u, p)-A_{i, p_{\beta}^{j}}^{\alpha}(\xi, u, \tilde{p})\right| \leq C\left(1+|p|^{2}+|\tilde{p}|^{2}\right)^{\frac{m-2}{2}} \omega(|p|,|p-\tilde{p}|) .
$$

(H2): $A_{i}^{\alpha}(\xi, u, p)$ satisfies the following ellipticity condition:

$$
A_{i, p_{\beta}^{j}}^{\alpha}(\xi, u, p) \eta_{i}^{\alpha} \eta_{j}^{\beta} \geq \lambda\left(1+|p|^{2}\right)^{\frac{m-2}{2}}|\eta|^{2}, \quad \forall \eta \in \mathbb{R}^{k \times N},
$$

where $\lambda$ is a positive constant.

(H3): There exists a modulus of continuity $\mu:(0, \infty) \rightarrow[0, \infty)$ such that

$$
\left|A_{i}^{\alpha}(\xi, u, p)-A_{i}^{\alpha}(\tilde{\xi}, \tilde{u}, p)\right| \leq K(|u|) \mu\left(\left(d^{m}(\xi, \tilde{\xi})+|u-\tilde{u}|^{m}\right)^{\frac{1}{m}}\right)(1+|p|)^{\frac{m}{2}}
$$

where $K(\cdot):[0, \infty) \rightarrow[0, \infty)$ is monotonously nondecreasing. Without loss of generality, we assume $K(\cdot) \geq 1$ and that $(\mu 1) \mu$ is nondecreasing with $\mu(0+)=0 \mu(1)=1 ;(\mu 2) \mu$ is concave, and $r \rightarrow r^{-\gamma} \mu(r)$ is nonincreasing for some exponent $\gamma \in(0,1)$; ( $\left.\mu 3\right)$ Dini condition $H(r)=\int_{0}^{r} \frac{\sqrt{\mu(\rho)}}{\rho} d \rho<\infty$ for some $r>0$.

(H4) (Controllable growth condition): The term $B^{\alpha}$ satisfies sub-quadratic controllable growth condition

$$
\left|B^{\alpha}(\xi, u, p)\right| \leq C\left(1+|u|^{r-1}+|p|^{m(r-1) / r}\right), \quad 1<m<2,
$$

where $C$ is a positive constant, and $r=\frac{m Q}{Q-m}$ if $m<Q$; or $Q \leq r<+\infty$ if $m=Q$. We note that $Q \geq 3$ is the homogeneous dimension in non-Abelian Carnot groups (see (2.3) below), and the exponent $m$ satisfies $1<m<2$. So those infer that $m<Q$, and then $r=\frac{m Q}{Q-m}$ in our setting.

We follow the strategy in the Euclidean case used by Duzaar et al. in [14] and Duzaar and Grotowski in [8] with the necessary modification to handle the sub-elliptic case in Carnot groups. First, inspired by [18], we choose horizontal variables to construct an auxiliary function to establish Caccioppoli type inequality; see Lemma 7 below. The method of using Taylor's expansion in [14] cannot be easily adapted to our situation. Instead, we choose different auxiliary functions and apply the Sobolev-Poincaré type inequality (3.6) to obtain the desired excess improvement estimates.

The main result in this paper is as follows.

Theorem 1 Assume that coefficients $A_{i}^{\alpha}$ and $B^{\alpha}$ satisfy conditions $(\mathrm{H} 1)-(\mathrm{H} 4)$ with $(\mu 1)-$ ( $\mu 3)$, and $u \in H W^{1, m}\left(\Omega, \mathbb{R}^{N}\right)$ is a weak solution to (1.3) with bounded domain $\Omega \subset G$. Then there exists an open subset $\Omega_{0} \subset \Omega$ such that $u \in C^{1}\left(\Omega_{0}, \mathbb{R}^{N}\right)$. Furthermore, the closed singular set $\Omega \backslash \Omega_{0} \subset \Sigma_{1} \cup \Sigma_{2}$ of Lebesgue measure zero, where

$$
\begin{aligned}
& \Sigma_{1}=\left\{\xi_{0} \in \Omega: \lim _{r \rightarrow 0^{+}} \sup \left(\left|(X u)_{\xi_{0}, r}\right|\right)=\infty\right\}, \\
& \Sigma_{2}=\left\{\xi_{0} \in \Omega: \lim _{r \rightarrow 0^{+}} \inf f_{B_{r}\left(\xi_{0}\right)}\left|X u-(X u)_{\xi_{0}, r}\right|^{m} d \xi>0\right\} .
\end{aligned}
$$


In addition, for $\tau \in[\gamma, 1)$ and $\xi_{0} \in \Omega \backslash \Omega_{0}$, the derivative $X u$ has the modulus of continuity $r \rightarrow r^{\tau}+M(r)$ in a neighborhood of $\xi_{0}$.

It is worth pointing out that the Haar measure in Carnot groups $\mathbb{G}$ with the underlying manifold $\mathbb{R}^{n}$ is just the Lebesgue measure in $\mathbb{R}^{n}$. Our result is optimal in the sense that when $\mu(\rho)=\rho^{\gamma}, 0<\gamma<1$, we have $M(r)=\int_{0}^{r} \frac{\mu(\rho)}{\rho} d \rho=\gamma^{-1} r^{\gamma}$, and $C^{1, \gamma}$ regularity is known to be optimal in that case; see the reference [2] by Wang and Liao.

\section{Preliminaries}

A Carnot group $G$ of step $r$ is a simply connected, nilpotent Lie group whose Lie algebra $\mathfrak{g}$ admits a stratification, i.e., $\mathfrak{g}=\bigoplus_{j=1}^{r} V^{j}$ such that $\left[V^{1}, V^{j}\right]=V^{j+1}, j=1, \ldots, r-1$ and $\left[V^{1}, V^{r}\right]=\{0\}$. Let $X_{i}^{l}$ be a left-invariant basis vector field of $V^{l}$ with $1 \leq l \leq r$ and $1 \leq i \leq m_{l}$, where $m_{l}$ is the dimension of $V^{l}$. For simplicity, we write $X_{i}=X_{i}^{1}, k=m_{1}$, and denote by $X=\left(X_{1}, \ldots, X_{k}\right)$ the horizontal gradient. We say that $X_{i}(i=1, \ldots, k)$ are the horizontal vector fields with the form

$$
X_{i}=\partial_{i}+\sum_{j=i+1}^{n} a_{i j}(\xi) \partial_{j}, \quad X_{i}(0)=\partial_{i},
$$

where $a_{i j}(\xi)$ is a polynomial.

We write

$$
\xi=\left(\xi^{1}, \xi^{2}, \ldots, \xi^{r}\right)=\left(x_{1}^{1}, x_{2}^{1}, \ldots, x_{m_{1}}^{1} ; x_{1}^{2}, \ldots, x_{m_{2}}^{2} ; \ldots ; x_{1}^{r}, \ldots, x_{m_{l}}^{r}\right) \in G
$$

and the distance from origin is defined by

$$
d(\xi)=\left[\sum_{l=1}^{r}\left(\sum_{i=1}^{m_{l}}\left|x_{i}^{l}\right|^{2}\right)^{\frac{r !}{l}}\right]^{\frac{1}{2 r !}}
$$

For any $\xi, \eta \in G$, we set $d(\xi, \eta)=d\left(\eta^{-1} \circ \xi\right)$, where $\eta^{-1}=-\eta=\left(-\eta^{1}, \ldots,-\eta^{r}\right)$ is the reverse of $\eta$, and $\circ$ is the multiplication rule in $G$ defined by $\xi \circ \widetilde{\xi}=\xi+\widetilde{\xi}+P(\xi, \widetilde{\xi}), \xi, \widetilde{\xi} \in G$, where $P: G \times G \mapsto G$ has polynomial components. Following [32], we denote by $\omega_{G}=\left|B_{1}(0)\right|_{G}$ the volume of unit ball. Then $\left|B_{r}(\xi)\right|_{G}=\omega_{G} r^{Q}$, where

$$
Q=\sum_{l=1}^{r} \operatorname{lm} l
$$

is the homogeneous dimension of $G$.

The non-Abelian Heisenberg group $\mathbb{H}^{n}$ is the simplest and the most important prototype of the Carnot group with step $r=2$, which is defined as $\mathbb{R}^{2 n+1}$ endowed with the following group multiplication:

$$
\left(\xi^{1}, t\right) \cdot\left(\tilde{\xi}^{1}, \tilde{t}\right):=\left(\xi^{1}+\tilde{\xi}^{1}, t+\tilde{t}+\frac{1}{2} \sum_{i=1}^{n}\left(x_{i} \tilde{y}_{i}-\tilde{x}_{i} y_{i}\right)\right) .
$$


The basic vector fields corresponding to its Lie algebra $\mathfrak{g}$ can be explicitly calculated by the exponential map and are given by

$$
X_{i}=\frac{\partial}{\partial x_{i}}-\frac{y_{i}}{2} \frac{\partial}{\partial t}, \quad X_{i+n}=\frac{\partial}{\partial y_{i}}+\frac{x_{i}}{2} \frac{\partial}{\partial t}, \quad T=\frac{\partial}{\partial t}
$$

for $i=1,2, \ldots, n$. The homogeneous dimension $Q$ in the Heisenberg group $H^{n}$ is $2 n+2 \geq 4$. Given the property of the Heisenberg algebra $\mathfrak{g}$, one has $\mathfrak{g}=\mathbb{R}^{2 n+1}=V_{1} \oplus V_{2}$ with $V_{1}=$ $\mathbb{R}^{2 n} \times\{0\}$ and $V_{2}=\{0\}_{\mathbb{R}^{2 n}} \times \mathbb{R}$ such that $\left[X_{i}, X_{n+j}\right]=T \delta_{i j}$ and $\left[V_{1}, V_{1}\right]=V_{2}$.

Let $1 \leq m<\infty$ and $\Omega \subset G$ be an open set. If $u \in L^{m}(\Omega)$ satisfies

$$
\|u\|_{H W^{1, m}(\Omega)}=\|u\|_{L^{m}(\Omega)}+\sum_{i=1}^{k}\left\|X_{i} u\right\|_{L^{m}(\Omega)}<\infty
$$

we say that $u$ belongs to the horizontal Sobolev space. The space $H W_{0}^{1, m}(\Omega)$ is the completion of $C_{0}^{\infty}(\Omega)$ under the norm (2.6).

Throughout the paper, we shall use the functions $V, W: \mathbb{R}^{n} \rightarrow \mathbb{R}^{n}$ defined by

$$
V(\varsigma)=\varsigma /\left(1+|\varsigma|^{2}\right)^{\frac{2-m}{4}}, \quad W(\varsigma)=\varsigma /\left(1+|\varsigma|^{2-m}\right)^{\frac{1}{2}}
$$

for each $\zeta \in \mathbb{R}^{n}$ and $m>1$. By the elementary inequality $\|x\|_{\frac{2}{2-m}} \leq\|x\|_{1} \leq 2^{\frac{m}{2}}\|x\|_{\frac{2}{2-m}}$ applied to the vector $x=\left(1,|\varsigma|^{2-m}\right) \in \mathbb{R}^{2}$, we conclude that

$$
\left(1+|\zeta|^{2}\right)^{\frac{2-m}{2}} \leq 1+|\zeta|^{2-m} \leq 2^{\frac{m}{2}}\left(1+|\zeta|^{2}\right)^{\frac{2-m}{2}},
$$

which immediately yields

$$
|W(\varsigma)| \leq|V(\varsigma)| \leq 2^{\frac{m}{4}}|W(\varsigma)| \text {. }
$$

The purpose of introducing $W$ is the fact that in contrast to $|V|^{\frac{2}{m}}$, the function $|W|^{\frac{2}{m}}$ is convex (see [9]).

The following lemma includes some useful properties of the function $V$, which can be found in Lemma 2.1 of [33].

Lemma 2 Let $m \in(1,2)$ and $V, W: \mathbb{R}^{n} \rightarrow \mathbb{R}^{n}$ be the functions defined in (2.7). Then there holds for any $\varsigma_{1}, \varsigma_{2} \in \mathbb{R}^{n}$ and $t>0$ :

(1) $\frac{1}{\sqrt{2}} \min \left(\left|\varsigma_{1}\right|,\left|\varsigma_{1}\right|^{\frac{m}{2}}\right) \leq\left|V\left(\varsigma_{1}\right)\right| \leq \min \left(\left|\varsigma_{1}\right|,\left|\varsigma_{1}\right|^{\frac{m}{2}}\right)$;

(2) $\left|V\left(t \varsigma_{1}\right)\right| \leq \max \left(t, t^{\frac{m}{2}}\right)\left|V\left(\varsigma_{1}\right)\right|$;

(3) $\left|V\left(\varsigma_{1}+\varsigma_{2}\right)\right| \leq C(m)\left(\left|V\left(\varsigma_{1}\right)\right|+\left|V\left(\varsigma_{2}\right)\right|\right)$;

(4) $\frac{m}{2}\left|\varsigma_{1}-\varsigma_{2}\right| \leq\left|V\left(\varsigma_{1}\right)-V\left(\varsigma_{2}\right)\right| /\left(1+\left|\varsigma_{1}\right|^{2}+\left|\varsigma_{2}\right|^{2}\right)^{\frac{m-2}{4}} \leq C(m, n)\left|\varsigma_{1}-\varsigma_{2}\right|$;

(5) $\left|V\left(\varsigma_{1}\right)-V\left(\varsigma_{2}\right)\right| \leq C(m, n)\left|V\left(\varsigma_{1}-\varsigma_{2}\right)\right|$;

(6) $\left|V\left(\varsigma_{1}-\varsigma_{2}\right)\right| \leq C(m, M)\left|V\left(\varsigma_{1}\right)-V\left(\varsigma_{2}\right)\right|$ for all $\varsigma_{1}$ with $\left|\varsigma_{2}\right| \leq M$.

The inequalities (1)-(3) also hold if $V$ is replaced by $W$.

Let $\varsigma_{1}, \varsigma_{2} \in \mathbb{R}^{n}$ with $\left|\varsigma_{2}\right| \leq M$, and we mention that the following two estimates can be deduced from Lemma 2(1) and (6):

$$
\left|\varsigma_{1}-\varsigma_{2}\right|^{2} \leq C(m, M)\left|V\left(\varsigma_{1}\right)-V\left(\varsigma_{2}\right)\right|^{2}
$$


for $\left|\varsigma_{1}-\varsigma_{2}\right| \leq 1$, while for $\left|\varsigma_{1}-\varsigma_{2}\right|>1$,

$$
\left|\varsigma_{1}-\varsigma_{2}\right|^{m} \leq C(m, M)\left|V\left(\varsigma_{1}\right)-V\left(\varsigma_{2}\right)\right|^{2} .
$$

The next lemma is an algebraic fact from [34].

Lemma 3 For every $t \in\left(-\frac{1}{2}, 0\right)$ and $\eta \geq 0$, it holds

$$
1 \leq \frac{\int_{0}^{1}\left(\eta^{2}+|p+s(\tilde{p}-p)|^{2}\right)^{t} d s}{\left(\eta^{2}+|p|^{2}+|\tilde{p}|^{2}\right)^{t}} \leq \frac{8}{2 t+1}
$$

for any $p, \tilde{p} \in \mathbb{R}^{k \times N}$, not both zero if $\eta=0$.

The nondecreasing property of $\mu$ yields $s \mu(t) \leq s \mu(t)$ for all $0 \leq t \leq s$. By the nonincreasing property of $r \mapsto \frac{\mu(r)}{r}$ and $\mu(1) \leq 1$, we conclude that

$$
s \mu(t) \leq s \mu(s)+t, \quad s \in[0,1], t>0 .
$$

By ( $\mu 2)$, we further obtain for $\theta \in(0,1), t>0, j \in \mathbb{N} \cup\{0\}$,

$$
\frac{2}{\gamma}\left(1-\theta^{\gamma}\right) \mu^{1 / 2}\left(\theta^{2 j} t\right)=\int_{\theta^{2(j+1)} t}^{\theta^{2 j} t} \tau^{\frac{\gamma}{2}-1} \frac{\mu^{1 / 2}\left(\theta^{2 j} t\right)}{\left(\theta^{2 j} t\right)^{\gamma / 2}} d \tau \leq \int_{\theta^{2(j+1)} t}^{\theta^{2 i} t} \frac{\mu^{1 / 2}(\tau)}{\tau} d \tau,
$$

which implies

$$
\sum_{j=0}^{\infty} \mu^{1 / 2}\left(\theta^{2 j} t\right) \leq \frac{\gamma}{2\left(1-\theta^{\gamma}\right)} H^{1 / 2}(t)
$$

It yields particularly that $\mu(t) \leq \frac{\gamma^{2}}{4} H(t)$ for all $t \leq 0$, and $t \mapsto t^{-\gamma} H(t)$ is also nonincreasing. In what follows, we denote $\rho_{1}(s, t)=(1+s+t)^{-1} K(s+t)^{-1}$, and $K_{1}(s, t)=(1+t)^{2 m} K^{4}(s+$ $t$ ) for $s, t \geq 0$, and note that $\rho_{1} \leq 1$ and that $s \rightarrow \rho_{1}(s, t), t \rightarrow \rho_{1}(s, t)$ are nonincreasing functions.

\section{Caccioppoli type inequality}

We first generalize an $\mathcal{A}$-harmonic approximation lemma in our setting. Then a SobolevPoincaré type inequality (Lemma 5) and a prior estimate (Lemma 6) are introduced for the sub-quadratic case in Carnot groups, and the detailed proofs can be found in [2] by Wang, Liao and $\mathrm{Yu}$. The last and key point is to prove a Caccioppoli type inequality.

$\operatorname{By} \operatorname{Bil}\left(\mathbb{R}^{k \times N}\right)$ we denote the collection of bi-linear forms defined in $\mathbb{R}^{k \times N}$. Let $\mathcal{A} \in$ $\operatorname{Bil}\left(\mathbb{R}^{k \times N}\right)$, we say that a function $h \in H W^{1, m}\left(\Omega, \mathbb{R}^{N}\right)$ is $\mathcal{A}$-harmonic if $h$ satisfies

$$
\int_{\Omega} \mathcal{A}(X h, X \varphi) d \xi=0, \quad \forall \varphi \in C_{0}^{1}\left(\Omega, \mathbb{R}^{N}\right)
$$

Similarly to [9], one can establish the following $\mathcal{A}$-harmonic approximation lemma for the case $1<m<2$ in Carnot groups. 
Lemma 4 Let $\lambda$ and $L$ be fixed positive numbers, $1<m<2$, and $k, N \in \mathbb{N}$ with $k \geq 2$. Suppose that for any given $\varepsilon>0$, there exists $\delta=\delta(k, N, \lambda, L, \varepsilon) \in(0,1]$ with the following properties:

(I) for any bi-linear form $\mathcal{A} \in \operatorname{Bil}\left(\mathbb{R}^{k \times N}\right)$ satisfying

$$
\mathcal{A}(v, v) \geq \lambda|v|^{2} \quad \text { and } \quad \mathcal{A}(v, \bar{v}) \leq L|v||\bar{v}|, \quad v, \bar{v} \in \mathbb{R}^{k \times N},
$$

(II) for any function $g \in H W^{1, m}\left(B_{\rho}\left(\xi_{0}\right), \mathbb{R}^{N}\right)$ satisfying

$$
\begin{aligned}
& f_{B_{\rho}\left(\xi_{0}\right)}|W(X g)|^{2} d \xi \leq \Upsilon^{2} \leq 1 \text { and } \\
& \left|f_{B_{\rho}\left(\xi_{0}\right)} \mathcal{A}(X g, X \varphi) d \xi\right| \leq \Upsilon \delta \sup _{B_{\rho}\left(\xi_{0}\right)}|X \varphi|, \quad \forall \varphi \in C_{0}^{1}\left(B_{\rho}\left(\xi_{0}\right), \mathbb{R}^{N}\right) .
\end{aligned}
$$

Then there is an $\mathcal{A}$-harmonic function

$$
h \in H=\left\{\left.h \in H W^{1, m}\left(B_{\rho}\left(\xi_{0}\right), \mathbb{R}^{N}\right)\left|f_{B_{\rho}\left(\xi_{0}\right)}\right| W(X h)\right|^{2} d \xi \leq 1\right\}
$$

such that

$$
f_{B_{\rho}\left(\xi_{0}\right)}\left|W\left(\frac{g-\Upsilon h}{\rho}\right)\right|^{2} d \xi \leq \Upsilon^{2} \varepsilon
$$

Lemma 5 (Sobolev-Poincaré type inequality) Let $m \in(1,2)$ and $u \in H W^{1, m}\left(B_{\rho}\left(\xi_{0}\right), \mathbb{R}^{N}\right)$ with $B_{\rho}\left(\xi_{0}\right) \subset \Omega$, then

$$
\left(f_{B_{\rho}\left(\xi_{0}\right)}\left|W\left(\frac{u-u_{\xi_{0}, \rho}}{\rho}\right)\right|^{\frac{2 Q}{Q-m}} d \xi\right)^{\frac{Q-m}{2 Q}} \leq C_{P}\left(f_{B_{\rho}\left(\xi_{0}\right)}|W(X u)|^{2} d \xi\right)^{\frac{1}{2}} .
$$

Furthermore, the analogous inequality is valid with $W$ replaced by $V$ defined in (2.7), and in particular, the inequality also holds if we substitute 2 for $\frac{2 Q}{Q-m}$.

Lemma 6 Let $u \in H W^{1,1}\left(\Omega, \mathbb{R}^{N}\right)$ be a weak solution of the systems with constant coefficients

$$
\int_{\Omega} A_{i j}^{\alpha \beta} X_{j} u^{\beta} X_{i} \phi^{\alpha} d \xi=0, \quad \phi \in C_{0}^{\infty}\left(\Omega, \mathbb{R}^{N}\right)
$$

Then $u$ is smooth and there exists $C_{0} \geq 1$ such that for any $B_{\rho}\left(\xi_{0}\right) \subset \Omega$,

$$
\sup _{B_{\rho / 2}\left(\xi_{0}\right)}\left(\left|u-u_{\xi_{0}, \rho}\right|^{2}+\rho^{2}|X u|^{2}+\rho^{4}\left|X^{2} u\right|^{2}\right) \leq C_{0} \rho^{2} f_{B_{\rho}\left(\xi_{0}\right)}|X u|^{2} d \xi
$$

To establish the main result, a crucial ingredient in the local regularity of weak solutions to systems (1.3) is a Caccioppoli type inequality. We prove a version which is adapted to our situation. 
Lemma 7 (Caccioppoli type inequality) Let $u \in H W^{1, m}\left(\Omega, \mathbb{R}^{N}\right)$ be a weak solution to system (1.3) under conditions (H1)-(H4) with $(\mu 1)-(\mu 3)$. Then, for any $\xi_{0}=\left(\xi_{0}^{1}, \ldots, \xi_{0}^{r}\right) \in \Omega$, and $0<t<s \leq \rho_{1}^{\frac{m}{(2-m)(m-1)}}\left(\left|u_{0}\right|,\left|p_{0}\right|\right)$, it holds

$$
f_{B_{t}\left(\xi_{0}\right)}\left|V\left(X u-p_{0}\right)\right|^{2} d \xi \leq C_{c}\left(f_{B_{s}\left(\xi_{0}\right)}\left|V\left(\frac{u-u_{0}-p_{0}\left(\xi^{1}-\xi_{0}^{1}\right)}{s-t}\right)\right|^{2} d \xi+U\right)
$$

with

$$
\begin{aligned}
U= & {\left[K(\cdot)\left(1+\left|p_{0}\right|\right)\right]^{2 m /(m-1)} \mu^{2}\left(s^{(2-m)(m-1) / m}\right)+\left(1+2 M+\left|p_{0}\right| s\right)^{m /(m-1)^{2}} s^{m-1} } \\
& +\mu^{2}\left(s^{(2-m)(m-1) / m}\right)\left[f_{B_{s}\left(\xi_{0}\right)}\left(|X u|^{m}+|u|^{r}+1\right) d \xi\right]^{\left(1-\frac{1}{r}\right) \frac{m}{m-1}}
\end{aligned}
$$

where we denote $K(\cdot)=K\left(\left|u_{0}\right|+\left|p_{0}\right|\right), C_{c}=C_{c}(Q, N, m, L, \lambda, M)$, and $\xi^{1}=\left(\xi_{1}^{1}, \xi_{2}^{1}, \ldots, \xi_{k}^{1}\right)$ is the horizontal component of $\xi \in G$.

Proof Let $\eta \in C_{0}^{\infty}\left(B_{s}\left(\xi_{0}\right)\right)$ be a standard cut-off function satisfying $0 \leq \eta \leq 1,|X \eta|<\frac{C}{s-t}$ and $\eta \equiv 1$ on $B_{t}\left(\xi_{0}\right)$. Inspired by the way of [18], we let $v=u(\xi)-u_{0}-p_{0}\left(\xi^{1}-\xi_{0}^{1}\right)$, and then define two functions

$$
\varphi=\eta \nu, \quad \phi=(1-\eta) \nu,
$$

one has

$$
X \varphi+X \phi=X u-p_{0}
$$

and

$$
|V(X \varphi)|,|V(X \phi)| \leq C(m)\left(|V(X v)|+\left|V\left(\frac{v}{s-t}\right)\right|\right) .
$$

Using hypothesis (H2), Lemma 3 and the elementary inequality

$$
1+|a|^{2}+|b-a|^{2} \leq 3\left(1+|a|^{2}+|b|^{2}\right),
$$

we have

$$
\begin{aligned}
& \int_{B_{s}\left(\xi_{0}\right)}\left[A_{i}^{\alpha}\left(\xi, u, p_{0}+X \varphi\right)-A_{i}^{\alpha}\left(\xi, u, p_{0}\right)\right] X_{i} \varphi^{\alpha} d \xi \\
& =\int_{B_{s}\left(\xi_{0}\right)} \int_{0}^{1} \frac{\partial A_{i}^{\alpha}\left(\xi, u, p_{0}+\theta X \varphi\right)}{\partial p_{j}^{\beta}} d \theta X_{j} \varphi^{\beta} X_{i} \varphi^{\alpha} d \xi \\
& \geq \lambda \int_{B_{s}\left(\xi_{0}\right)}\left[\int_{0}^{1}\left(1+\left|p_{0}+\theta X \varphi\right|^{2}\right)^{(m-2) / 2} d \theta\right]|X \varphi|^{2} d \xi \\
& \geq 3^{(m-2) / 2} \lambda \int_{B_{s}\left(\xi_{0}\right)}\left(1+\left|p_{0}\right|^{2}+|X \varphi|^{2}\right)^{(m-2) / 2}|X \varphi|^{2} d \xi .
\end{aligned}
$$


From (3.15), it follows that

$$
\begin{aligned}
& 3^{(m-2) / 2} \lambda \int_{B_{s}\left(\xi_{0}\right)}\left(1+\left|p_{0}\right|^{2}+|X \varphi|^{2}\right)^{(m-2) / 2}|X \varphi|^{2} d \xi \\
& \leq \int_{B_{s}\left(\xi_{0}\right)}\left[A_{i}^{\alpha}\left(\xi, u, p_{0}+X \varphi\right)-A_{i}^{\alpha}\left(\xi, u, p_{0}\right)\right] X_{i} \varphi^{\alpha} d \xi \\
& \leq-\int_{B_{s}\left(\xi_{0}\right)}\left[A_{i}^{\alpha}\left(\xi, u, p_{0}\right)-A_{i}^{\alpha}\left(\xi, u_{0}+p_{0}\left(\xi^{1}-\xi_{0}^{1}\right), p_{0}\right)\right] X_{i} \varphi^{\alpha} d \xi \\
&-\int_{B_{s}\left(\xi_{0}\right)}\left[A_{i}^{\alpha}\left(\xi, u_{0}+p_{0}\left(\xi^{1}-\xi_{0}^{1}\right), p_{0}\right)-A_{i}^{\alpha}\left(\xi_{0}, u_{0}, p_{0}\right)\right] X_{i} \varphi^{\alpha} d \xi \\
&-\int_{B_{s}\left(\xi_{0}\right)} \int_{0}^{1} \frac{\partial A_{i}^{\alpha}\left(\xi, u, X u-\theta\left(X u-X \varphi-p_{0}\right)\right)}{\partial p_{j}^{\beta}} d \theta X_{j} \phi^{\beta} X_{i} \varphi^{\alpha} d \xi \\
&+\int_{B_{s}\left(\xi_{0}\right)} B_{i}(\xi, u, X u) \varphi^{\alpha} d \xi \\
&:= I+I I+I I I+I V,
\end{aligned}
$$

where we have used (3.12), (1.3) and the fact that

$$
\int_{B_{S}\left(\xi_{0}\right)} A_{i}^{\alpha}\left(\xi_{0}, u_{0}, p_{0}\right) X \varphi^{\alpha} d \xi=0
$$

Noting that $\varphi=v$ on $B_{t}\left(\xi_{0}\right), m-2<0$ and (3.14), the left-hand side in (3.16) can be estimated by

$$
\begin{aligned}
& 3^{(m-2) / 2} \lambda \int_{B_{t}\left(\xi_{0}\right)}\left(1+\left|p_{0}\right|^{2}+|X \varphi|^{2}\right)^{(m-2) / 2}|X \varphi|^{2} d \xi \\
& \quad=3^{(m-2) / 2} \lambda \int_{B_{t}\left(\xi_{0}\right)}\left(1+\left|p_{0}\right|^{2}+|X \nu|^{2}\right)^{(m-2) / 2}|X \nu|^{2} d \xi \\
& \geq C(m) \lambda \int_{B_{t}\left(\xi_{0}\right)}\left[\left(1+\left|p_{0}\right|^{2}+|X u|^{2}\right)^{(m-2) / 4}\left|X u-p_{0}\right|\right]^{2} d \xi \\
& \geq C(k, N, m, \lambda) \int_{B_{t}\left(\xi_{0}\right)}\left|V(X u)-V\left(p_{0}\right)\right|^{2} d \xi \\
& \geq C(k, N, m, \lambda, M) \int_{B_{t}\left(\xi_{0}\right)}|V(X v)|^{2} d \xi
\end{aligned}
$$

where we have applied Lemma 2(4) in the second inequality and Lemma 2(6) for the final inequality.

The structure condition $(\mathrm{H} 3)$ yields

$$
\begin{aligned}
I & \leq \int_{B_{s}\left(\xi_{0}\right)} K(\cdot)\left(1+\left|p_{0}\right|\right)^{m / 2} \mu(|v|)|X \varphi| d \xi \\
& \leq \frac{1}{s^{(2-m)(m-1) / m}} \int_{B_{s}\left(\xi_{0}\right)}\left[K(\cdot)\left(1+\left|p_{0}\right|\right)^{m / 2} s^{(2-m)(m-1) / m} \mu(|v|)\right]|X \varphi| d \xi \\
& \leq \frac{1}{s^{(2-m)(m-1) / m}} \int_{B_{S}\left(\xi_{0}\right)}\left[K(\cdot)\left(1+\left|p_{0}\right|\right)^{m / 2} s^{(2-m)(m-1) / m}\right.
\end{aligned}
$$




$$
\begin{aligned}
& \left.\times \mu\left(K(\cdot)\left(1+\left|p_{0}\right|\right)^{m / 2} s^{(2-m)(m-1) / m}\right)+|v|\right]|X \varphi| d \xi \\
\leq & \frac{1}{s^{(2-m)(m-1) / m}} \int_{B_{s}\left(\xi_{0}\right)}\left[K^{2}(\cdot)\left(1+\left|p_{0}\right|\right)^{m} s^{(2-m)(m-1) / m} \mu\left(s^{(2-m)(m-1) / m}\right)+|v|\right]|X \varphi| d \xi \\
\leq & \int_{B_{s}\left(\xi_{0}\right)}\left[K^{2}(\cdot)\left(1+\left|p_{0}\right|\right)^{m} \mu\left(s^{(2-m)(m-1) / m}\right)+\frac{|v|}{s^{(2-m)(m-1) / m}}\right]|X \varphi| d \xi
\end{aligned}
$$

where we have used the inequality $s \mu(t) \leq s \mu(s)+t$ for $s \in[0,1]$ and $t>0$.

To obtain a suitable estimate for $I$, we need to take the domain $B_{s}\left(\xi_{0}\right)$ into four parts: $B_{s}\left(\xi_{0}\right) \cap\{|v / s|>1\} \cap\{|X \varphi| \leq 1\}, B_{s}\left(\xi_{0}\right) \cap\{|v / s|>1\} \cap\{|X \varphi|>1\}, B_{s}\left(\xi_{0}\right) \cap\{|v / s| \leq 1\} \cap$ $\{|X \varphi|>1\}, B_{s}\left(\xi_{0}\right) \cap\{|v / s| \leq 1\} \cap\{|X \varphi| \leq 1\}$, and we will use Young's inequality, (2.10) and (2.11), repeatedly.

Case 1: On the part $B_{s}\left(\xi_{0}\right) \cap\{|v / s|>1\} \cap\{|X \varphi|>1\}$, we see

$$
\begin{aligned}
& {\left[K^{2}(\cdot)\left(1+\left|p_{0}\right|\right)^{m} \mu\left(s^{(2-m)(m-1) / m}\right)+\frac{|v|}{s^{(2-m)(m-1) / m}}\right]|X \varphi|} \\
& \leq 2 \varepsilon|X \varphi|^{m}+\varepsilon^{-1}\left[K^{2}(\cdot)\left(1+\left|p_{0}\right|\right)^{m} \mu\left(s^{(2-m)(m-1) / m}\right)\right]^{m /(m-1)}+\varepsilon^{-1}\left|\frac{v}{s}\right||v|^{1 /(m-1)} s^{m-1} \\
& \leq 2 \varepsilon|X \varphi|^{m}+\varepsilon^{-1}\left[K^{2}(\cdot)\left(1+\left|p_{0}\right|\right)^{m} \mu\left(s^{(2-m)(m-1) / m}\right)\right]^{m /(m-1)} \\
& +\varepsilon^{-1}\left|\frac{v}{s}\right|^{m}+\varepsilon^{-1}|v|^{m /(m-1)^{2}} s^{m} \\
& \leq 2 \varepsilon C(m, M)|V(X \varphi)|^{2}+\varepsilon^{-1} C(m, M)\left|V\left(\frac{v}{s}\right)\right|^{2} \\
& +\varepsilon^{-1}\left[K^{2}(\cdot)\left(1+\left|p_{0}\right|\right)^{m} \mu\left(s^{(2-m)(m-1) / m}\right)\right]^{m /(m-1)}+\varepsilon^{-1}\left|2 M+p_{0} s\right|^{m /(m-1)^{2}} s^{m} .
\end{aligned}
$$

Case 2: On the set $B_{s}\left(\xi_{0}\right) \cap\{|v / s|>1\} \cap\{|X \varphi| \leq 1\}$, it holds

$$
\begin{aligned}
& {\left[K^{2}(\cdot)\left(1+\left|p_{0}\right|\right)^{m} \mu\left(s^{(2-m)(m-1) / m}\right)+\frac{|\nu|}{s^{(2-m)(m-1) / m}}\right]|X \varphi|} \\
& \quad \leq 2 \varepsilon|X \varphi|^{2}+\varepsilon^{-1}\left[K^{2}(\cdot)\left(1+\left|p_{0}\right|\right)^{m} \mu\left(s^{(2-m)(m-1) / m}\right)\right]^{2}+\varepsilon^{-1}\left|\frac{v}{s}\right|^{m}+\varepsilon^{-1}|\nu|^{m /(m-1)} s^{m} \\
& \leq 2 \varepsilon C(m, M)|V(X \varphi)|^{2}+\varepsilon^{-1} C(m, M)\left|V\left(\frac{v}{s}\right)\right|^{2} \\
& \quad+\varepsilon^{-1}\left[K^{2}(\cdot)\left(1+\left|p_{0}\right|\right)^{m} \mu\left(s^{(2-m)(m-1) / m}\right)\right]^{2}+\varepsilon^{-1}\left|2 M+p_{0} s\right|^{m /(m-1)} s^{m}
\end{aligned}
$$

where we have used the inequality $2(m-1) / m<1$.

Case 3: On the set $B_{s}\left(\xi_{0}\right) \cap\{|v / s| \leq 1\} \cap\{|X \varphi|>1\}$, observing $m /(m-1)>2$, one has

$$
\begin{aligned}
& {\left[K^{2}(\cdot)\left(1+\left|p_{0}\right|\right)^{m} \mu\left(s^{(2-m)(m-1) / m}\right)+\frac{|v|}{s^{(2-m)(m-1) / m}}\right]|X \varphi|} \\
& \leq 2 \varepsilon|X \varphi|^{m}+\varepsilon^{-1}\left[K^{2}(\cdot)\left(1+\left|p_{0}\right|\right)^{m} \mu\left(s^{(2-m)(m-1) / m}\right)\right]^{m /(m-1)}+\varepsilon^{-1}\left|\frac{v}{s}\right||v|^{1 /(m-1)} s^{m-1} \\
& \leq 2 \varepsilon C(m, M)|V(X \varphi)|^{2}+\varepsilon^{-1}\left[K^{2}(\cdot)\left(1+\left|p_{0}\right|\right)^{m} \mu\left(s^{(2-m)(m-1) / m}\right)\right]^{m /(m-1)} \\
& \quad+\varepsilon^{-1}\left|2 M+p_{0} s\right|^{1 /(m-1)} s^{m-1} .
\end{aligned}
$$


Case 4: For $B_{s}\left(\xi_{0}\right) \cap\{|v / s| \leq 1\} \cap\{|X \varphi| \leq 1\}$, there is

$$
\begin{aligned}
& {\left[K^{2}(\cdot)\left(1+\left|p_{0}\right|\right)^{m} \mu\left(s^{(2-m)(m-1) / m}\right)+\frac{|v|}{s^{(2-m)(m-1) / m}}\right]|X \varphi|} \\
& \quad \leq 2 \varepsilon C(m, M)|V(X \varphi)|^{2}+\varepsilon^{-1}\left[K^{2}(\cdot)\left(1+\left|p_{0}\right|\right)^{m} \mu\left(s^{(2-m)(m-1) / m}\right)\right]^{2}+\varepsilon^{-1} s^{m} .
\end{aligned}
$$

Combining these estimations with (3.18), we get

$$
\begin{aligned}
I \leq & \varepsilon C(m, M) \int_{B_{s}\left(\xi_{0}\right)}|V(X v)|^{2} d \xi+C(\varepsilon, m, M) \int_{B_{s}\left(\xi_{0}\right)}\left|V\left(\frac{v}{s-t}\right)\right|^{2} d \xi \\
& +\varepsilon^{-1} K^{2 m /(m-1)}(\cdot)\left(1+\left|p_{0}\right|\right)^{m^{2} /(m-1)} \mu^{2}\left(s^{(2-m)(m-1) / m}\right)\left|B_{s}\left(\xi_{0}\right)\right|_{G} \\
& +\varepsilon^{-1}\left(1+2 M+\left|p_{0}\right| s\right)^{m /(m-1)^{2}}\left|B_{s}\left(\xi_{0}\right)\right|_{G} s^{m-1} .
\end{aligned}
$$

Similarly to $I$, it follows that

$$
\begin{aligned}
I I \leq & \int_{B_{s}\left(\xi_{0}\right)} K(\cdot)\left(1+\left|p_{0}\right|\right)^{(m+2) / 2} \mu(s)|X \varphi| d \xi \\
\leq & \int_{B_{1}\left(\xi_{0}\right)}\left[\varepsilon|X \varphi|^{m}+\varepsilon^{-1} K^{m /(m-1)}(\cdot)\left(1+\left|p_{0}\right|\right)^{m(m+2) / 2(m-1)} \mu^{m /(m-1)}(s)\right] d \xi \\
& +\int_{B_{2}\left(\xi_{0}\right)}\left[\varepsilon|X \varphi|^{2}+\varepsilon^{-1} K^{2}(\cdot)\left(1+\left|p_{0}\right|\right)^{(m+2)} \mu^{2}(s)\right] d \xi \\
\leq & 2 \varepsilon C(m, M) \int_{B_{s}\left(\xi_{0}\right)}|V(X v)|^{2} d \xi+2 \varepsilon C(m, M) \int_{B_{s}\left(\xi_{0}\right)}\left|V\left(\frac{v}{s-t}\right)\right|^{2} d \xi \\
& +\varepsilon^{-1} K^{m /(m-1)}(\cdot)\left(1+\left|p_{0}\right|\right)^{m(m+2) / 2(m-1)} \mu^{2}(s)\left|B_{s}\left(\xi_{0}\right)\right|_{G} \cdot
\end{aligned}
$$

By (H1), Lemma 3 and (3.14), it holds

$$
\begin{aligned}
I I I & \leq L \int_{B_{s}\left(\xi_{0}\right)}\left[\int_{0}^{1}\left(1+|X u+\theta[(X u-X \phi)-X u]|^{2}\right)^{(m-2) / 2} d \theta\right]|X \phi||X \varphi| d \xi \\
& \leq \frac{8 L}{m-1} \int_{B_{s}\left(\xi_{0}\right)}\left(1+|X u|^{2}+|X u-X \phi|^{2}\right)^{(m-2) / 2}|X \phi||X \varphi| d \xi \\
& \leq \frac{8 L}{m-1} \int_{B_{s}\left(\xi_{0}\right)}\left(1+|X \phi|^{2}\right)^{(m-2) / 2}|X \phi||X \varphi| d \xi .
\end{aligned}
$$

Noting that $X \phi \subset B_{s} \backslash B_{t}$ and $-1 / 2<(m-2) / 2<0$, we split the domain $B_{s}\left(\xi_{0}\right)$ into four parts: $B_{s}\left(\xi_{0}\right) \cap\{|X \phi|>1\} \cap\{|X \varphi|>1\}, B_{s}\left(\xi_{0}\right) \cap\{|X \phi| \leq 1\} \cap\{|X \varphi| \leq 1\}, B_{s}\left(\xi_{0}\right) \cap\{|X \phi|>1\} \cap$ $\{|X \varphi| \leq 1\}$ and $B_{s}\left(\xi_{0}\right) \cap\{|X \phi| \leq 1\} \cap\{|X \varphi|>1\}$. Similarly to $I$, it follows that

$$
I I I \leq \frac{C(L, m, M)}{m-1}\left(\int_{B_{s}\left(\xi_{0}\right) \backslash B_{t}\left(\xi_{0}\right)}|V(X v)|^{2} d \xi+\int_{B_{s}\left(\xi_{0}\right)}\left|V\left(\frac{v}{s-t}\right)\right|^{2} d \xi\right) .
$$


Using Hölder's inequality, one has

$$
\begin{aligned}
I V & \leq C \int_{B_{S}\left(\xi_{0}\right)}\left(|X u|^{m}+|u|^{r}+1\right)^{1-\frac{1}{r}}|\varphi| d \xi \\
& \leq C\left[\int_{B_{S}\left(\xi_{0}\right)}\left(|X u|^{m}+|u|^{r}+1\right) d \xi\right]^{1-\frac{1}{r}}\left(\int_{B_{S}\left(\xi_{0}\right)}|\varphi|^{r} d \xi\right)^{\frac{1}{r}} .
\end{aligned}
$$

Analogously to $I$, we take the domain $B_{S}\left(\xi_{0}\right)$ into two parts.

Case 1: For $B_{s}\left(\xi_{0}\right) \cap\{|X v|>1\}$, by Sobolev type inequality, Young's inequality and (2.11), it follows that

$$
\begin{aligned}
& {\left[\int_{B_{s}\left(\xi_{0}\right)}\left(|X u|^{m}+|u|^{r}+1\right) d \xi\right]^{1-\frac{1}{r}}\left(\int_{B_{s}\left(\xi_{0}\right)}|\varphi|^{r} d \xi\right)^{\frac{1}{r}}} \\
& \leq C\left[\int_{B_{s}\left(\xi_{0}\right)}\left(|X u|^{m}+|u|^{r}+1\right) d \xi\right]^{1-\frac{1}{r}}\left(\int_{B_{s}\left(\xi_{0}\right)}|X v|^{m} d \xi\right)^{\frac{1}{m}} \\
& \leq C(\varepsilon)\left[\int_{B_{s}\left(\xi_{0}\right)}\left(|X u|^{m}+|u|^{r}+1\right) d \xi\right]^{\left(1-\frac{1}{r}\right) \frac{m}{m-1}}+C \varepsilon\left(\int_{B_{S}\left(\xi_{0}\right)}|X v|^{m} d \xi\right) \\
& \leq C(\varepsilon)\left[\int_{B_{s}\left(\xi_{0}\right)}\left(|X u|^{m}+|u|^{r}+1\right) d \xi\right]^{\left(1-\frac{1}{r}\right) \frac{m}{m-1}} \\
& \quad+C(m, M) \varepsilon\left(\int_{B_{s}\left(\xi_{0}\right)}|V(X v)|^{2} d \xi\right)
\end{aligned}
$$

where we have used $|\varphi|^{r}<|v|^{r}$.

Case 2: On the set $B_{s}\left(\xi_{0}\right) \cap\{|X v| \leq 1\}$,

$$
\begin{aligned}
& {\left[\int_{B_{S}\left(\xi_{0}\right)}\left(|X u|^{m}+|u|^{r}+1\right) d \xi\right]^{1-\frac{1}{r}}\left(\int_{B_{S}\left(\xi_{0}\right)}|\varphi|^{r} d \xi\right)^{\frac{1}{r}}} \\
& \quad \leq C(\varepsilon)\left[\int_{B_{S}\left(\xi_{0}\right)}\left(|X u|^{m}+|u|^{r}+1\right) d \xi\right]^{2\left(1-\frac{1}{r}\right)}+C(m, M) \varepsilon\left(\int_{B_{S}\left(\xi_{0}\right)}|V(X v)|^{2} d \xi\right),
\end{aligned}
$$

where we have used (2.10).

Combining these estimates in $I V$, we have

$$
\begin{aligned}
I V \leq & C(m, M) \varepsilon\left(\int_{B_{s}\left(\xi_{0}\right)}|V(X v)|^{2} d \xi\right)+C(\varepsilon)\left[\int_{B_{s}\left(\xi_{0}\right)}\left(|X u|^{m}+|u|^{r}+1\right) d \xi\right]^{\left(1-\frac{1}{r}\right) \frac{m}{m-1}} \\
& +C(\varepsilon)\left[\int_{B_{S}\left(\xi_{0}\right)}\left(|X u|^{m}+|u|^{r}+1\right) d \xi\right]^{2\left(1-\frac{1}{r}\right)} \cdot
\end{aligned}
$$

Substituting (3.17), (3.23), (3.24), (3.26) and (3.30) into (3.16), we finally arrive at

$$
\begin{aligned}
& {\left[3^{(m-2)} \lambda C^{2}(m, M)+\frac{C(L, m, M)}{m-1}\right] \int_{B_{t}\left(\xi_{0}\right)}|V(X v)|^{2} d \xi} \\
& \leq\left[\frac{C(L, m, M)}{m-1}+4 \varepsilon C(m, M)\right] \int_{B_{s}\left(\xi_{0}\right)}|V(X v)|^{2} d \xi
\end{aligned}
$$




$$
\begin{aligned}
& +C(\varepsilon, L, m, M) \int_{B_{s}\left(\xi_{0}\right)}\left|V\left(\frac{v}{s-t}\right)\right|^{2} d \xi \\
& +C(\varepsilon)\left[K(\cdot)\left(1+\left|p_{0}\right|\right)\right]^{2 m /(m-1)} \mu^{2}\left(s^{(2-m)(m-1) / m}\right)\left|B_{S}\left(\xi_{0}\right)\right|_{G} \\
& +C(\varepsilon)\left(1+2 M+\left|p_{0}\right| s\right)^{m /(m-1)^{2}}\left|B_{s}\left(\xi_{0}\right)\right|_{G} s^{m-1} \\
& +C(\varepsilon)\left[\int_{B_{s}\left(\xi_{0}\right)}\left(|X u|^{m}+|u|^{r}+1\right) d \xi\right]^{\left(1-\frac{1}{r}\right) \frac{m}{m-1}} \\
& +C(\varepsilon)\left[\int_{B_{s}\left(\xi_{0}\right)}\left(|X u|^{m}+|u|^{r}+1\right) d \xi\right]^{2\left(1-\frac{1}{r}\right)} .
\end{aligned}
$$

We let $\varepsilon=\left[3^{m-2} \lambda C(m, M)\right] / 5$. 'Filling the hole' with $\theta=\frac{\frac{C(L, m, M)}{m-1}+4 \varepsilon C(m, M)}{\frac{C(L, m, M)}{m-1}+3^{m-2} \lambda C^{2}(m, M)}<1$ yields

$$
\begin{aligned}
\int_{B_{t}\left(\xi_{0}\right)}\left|V\left(X u-p_{0}\right)\right|^{2} d \xi \leq & C\left(\int_{B_{s}\left(\xi_{0}\right)}\left|V\left(\frac{v}{s-t}\right)\right|^{2} d \xi+\hat{U}\right) \\
& +\theta \int_{B_{s}\left(\xi_{0}\right)}\left|V\left(X u-p_{0}\right)\right|^{2} d \xi
\end{aligned}
$$

where $C=C(N, L, \lambda, m, M)$, and

$$
\begin{aligned}
\hat{U}= & {\left[K(\cdot)\left(1+\left|p_{0}\right|\right)\right]^{2 m /(m-1)} \mu^{2}\left(s^{(2-m)(m-1) / m}\right)\left|B_{s}\left(\xi_{0}\right)\right|_{G} } \\
& +\left(1+2 M+\left|p_{0}\right| s\right)^{m /(m-1)^{2}}\left|B_{s}\left(\xi_{0}\right)\right|_{G} s^{m-1} \\
& +\left[\int_{B_{s}\left(\xi_{0}\right)}\left(|X u|^{m}+|u|^{r}+1\right) d \xi\right]^{\left(1-\frac{1}{r}\right) \frac{m}{m-1}} \\
& +\left[\int_{B_{s}\left(\xi_{0}\right)}\left(|X u|^{m}+|u|^{r}+1\right) d \xi\right]^{2\left(1-\frac{1}{r}\right)} .
\end{aligned}
$$

The proof is completed by taking mean values of integral and noting that $[m(r-1) / r(m-$ 1) -1$] Q=m /(m-1)$ and $s^{m /(m-1)} \leq s^{2(2-m)(m-1) / m} \leq \mu^{2}\left(s^{(2-m)(m-1) / m}\right)$.

\section{Proof of the main result}

This section will proceed to the proof of Theorem 1 by Lemmas 8-9. We first give a linearization strategy for nonlinear systems.

Lemma 8 We claim that if $\rho \leq \rho_{1}^{\frac{m}{(2-m)(m-1)}}\left(\left|u_{0}\right|,\left|p_{0}\right|\right)$ and $\varphi \in C_{0}^{\infty}\left(B_{\rho}\left(\xi_{0}\right), \mathbb{R}^{N}\right)$ with $\sup _{B_{\rho}\left(\xi_{0}\right)}|X \varphi| \leq 1$, then there exist some constants $C_{1}=C_{1}\left(L, m, M, C_{P}, K\right)>1$ such that

$$
\begin{aligned}
& f_{B_{\rho}\left(\xi_{0}\right)} A_{i, p_{\beta}^{j}}^{\alpha}\left(\xi_{0}, u_{0}, p_{0}\right)\left(X u-p_{0}\right) X \varphi^{\alpha} d \xi \\
& \leq C_{1} \sup _{B_{\rho}\left(\xi_{0}\right)}|X \varphi|\left[\omega^{\frac{1}{2}}\left(\left|p_{0}\right|, \Phi^{\frac{1}{2}}\left(\xi_{0}, \rho, p_{0}\right)\right) \Phi^{\frac{1}{2}}\left(\xi_{0}, \rho, p_{0}\right)\right. \\
& \left.\quad+\Phi\left(\xi_{0}, \rho, p_{0}\right)+\mu(\sqrt{\rho}) F\left(\left|u_{0}\right|,\left|p_{0}\right|\right)\right],
\end{aligned}
$$

where we denote $F(s, t)=K^{4 /(2-m)}(s+t)(2+t)^{2}+(1+s+t)^{r-1}$. 
Proof Noting the fact

$$
\begin{aligned}
& \int_{B_{\rho}\left(\xi_{0}\right)}\left[\int_{0}^{1} A_{i, p_{\beta}^{j}}^{\alpha}\left(\xi_{0}, u_{0}, \theta X u+(1-\theta) p_{0}\right)\left(X u-p_{0}\right) d \theta\right] X \varphi^{\alpha} d \xi \\
& =\int_{B_{\rho}\left(\xi_{0}\right)}\left[A_{i}^{\alpha}\left(\xi_{0}, u_{0}, X u\right)-A_{i}^{\alpha}\left(\xi_{0}, u_{0}, p_{0}\right)\right] X \varphi^{\alpha} d \xi \\
& =\int_{B_{\rho}\left(\xi_{0}\right)}\left[A_{i}^{\alpha}\left(\xi_{0}, u_{0}, X u\right)-A_{i}^{\alpha}(\xi, u, X u)\right] X \varphi^{\alpha} d \xi \\
& \quad+\int_{B_{\rho}\left(\xi_{0}\right)} B^{\alpha}(\xi, u, X u) \varphi^{\alpha} d \xi,
\end{aligned}
$$

we have

$$
\begin{aligned}
\int_{B_{\rho}\left(\xi_{0}\right)} & A_{i, p_{\beta}^{j}}^{\alpha}\left(\xi_{0}, u_{0}, p_{0}\right)\left(X u-p_{0}\right) X \varphi^{\alpha} d \xi \\
= & \int_{B_{\rho}\left(\xi_{0}\right)}\left[\int_{0}^{1} A_{i, p_{\beta}^{j}}^{\alpha}\left(\xi_{0}, u_{0}, p_{0}\right) d \theta\left(X u-p_{0}\right)\right] X \varphi^{\alpha} d \xi \\
\leq & \int_{B_{\rho}\left(\xi_{0}\right)}\left[\int_{0}^{1}\left|A_{i, p_{\beta}^{j}}^{\alpha}\left(\xi_{0}, u_{0}, p_{0}\right)-A_{i, p_{\beta}^{j}}^{\alpha}\left(\xi_{0}, u_{0}, \theta X u+(1-\theta) p_{0}\right)\right|\left|X u-p_{0}\right| d \theta\right] \\
& \times \sup _{B_{\rho}\left(\xi_{0}\right)}|X \varphi| d \xi \\
& +\int_{B_{\rho}\left(\xi_{0}\right)}\left|A_{i}^{\alpha}\left(\xi_{0}, u_{0}, X u\right)-A_{i}^{\alpha}\left(\xi, u_{0}+p_{0}\left(\xi^{1}-\xi_{0}^{1}\right), X u\right)\right| \sup _{B_{\rho}\left(\xi_{0}\right)}|X \varphi| d \xi \\
& +\int_{B_{\rho}\left(\xi_{0}\right)}\left|A_{i}^{\alpha}\left(\xi, u_{0}+p_{0}\left(\xi^{1}-\xi_{0}^{1}\right), X u\right)-A_{i}^{\alpha}(\xi, u, X u)\right| \sup _{B_{\rho}\left(\xi_{0}\right)}|X \varphi| d \xi \\
& +C \int_{B_{\rho}\left(\xi_{0}\right)}\left(|p|^{m\left(1-\frac{1}{r}\right)}+|u|^{r-1}+1\right)|\varphi| d \xi \\
:= & I^{\prime}+I I^{\prime}+I I I^{\prime}+I V^{\prime} .
\end{aligned}
$$

Using (H1) and estimate $(1.5)$ yields (note that $m-2<0$ )

$$
\begin{aligned}
I^{\prime} \leq & C(L) \sup _{B_{\rho}\left(\xi_{0}\right)}|X \varphi| \int_{B_{\rho}\left(\xi_{0}\right)}\left\{\int_{0}^{1}\left[\left(1+\left|p_{0}\right|^{2}\right)^{\frac{m-2}{2}}+\left(1+\left|p_{0}+\theta\left(X u-p_{0}\right)\right|^{2}\right)^{\frac{m-2}{2}}\right]^{\frac{1}{2}}\right. \\
& \left.\times\left[\left(1+\left|p_{0}\right|^{2}+\left|p_{0}+\theta\left(X u-p_{0}\right)\right|^{2}\right)^{\frac{m-2}{2}} \omega\left(\left|p_{0}\right|,\left|\theta\left(X u-p_{0}\right)\right|\right)\right]^{\frac{1}{2}} d \theta\right\}\left|X u-p_{0}\right| d \xi \\
\leq & C(L, m) \sup _{B_{\rho}\left(\xi_{0}\right)}|X \varphi| \int_{B_{\rho}\left(\xi_{0}\right)}\left(1+\left|X u-p_{0}\right|^{2}\right)^{\frac{m-2}{4}} \omega^{\frac{1}{2}}\left(\left|p_{0}\right|,\left|X u-p_{0}\right|\right)\left|X u-p_{0}\right| d \xi \\
\leq & C(L, m) \sup _{B_{\rho}\left(\xi_{0}\right)}|X \varphi| \int_{B_{\rho}\left(\xi_{0}\right)}\left(1+\left|X u-p_{0}\right|^{\frac{m-2}{2}}\right)\left|X u-p_{0}\right| \omega^{\frac{1}{2}}\left(\left|p_{0}\right|,\left|X u-p_{0}\right|\right) d \xi .
\end{aligned}
$$

Let

$$
B_{1}=: B_{\rho}\left(\xi_{0}\right) \cap\left\{\left|X u-p_{0}\right| \leq 1\right\}, \quad B_{2}=: B_{\rho}\left(\xi_{0}\right) \cap\left\{\left|X u-p_{0}\right|>1\right\} .
$$


Then it follows that, by first Hölder's inequality and then Jensen's inequality, we have

$$
\begin{aligned}
I^{\prime} \leq & C(L, m) \sup _{B_{\rho}\left(\xi_{0}\right)}|X \varphi|\left[\int_{B_{1}}\left|X u-p_{0}\right| \omega^{\frac{1}{2}}\left(\left|p_{0}\right|,\left|X u-p_{0}\right|\right) d \xi\right. \\
& \left.+\int_{B_{2}}\left|X u-p_{0}\right|^{\frac{m}{2}} \omega^{\frac{1}{2}}\left(\left|p_{0}\right|,\left|X u-p_{0}\right|\right) d \xi\right] \\
\leq & C(L, m) \sup _{B_{\rho}\left(\xi_{0}\right)}|X \varphi|\left(\int_{B_{1}}\left|X u-p_{0}\right|^{2} d \xi\right)^{\frac{1}{2}}\left(\int_{B_{\rho}\left(\xi_{0}\right)} \omega\left(\left|p_{0}\right|,\left|X u-p_{0}\right|\right) d \xi\right)^{\frac{1}{2}} \\
& +C(L, m) \sup _{B_{\rho}\left(\xi_{0}\right)}|X \varphi|\left(\int_{B_{2}}\left|X u-p_{0}\right|^{m} d \xi\right)^{\frac{1}{2}}\left(\int_{B_{\rho}\left(\xi_{0}\right)} \omega\left(\left|p_{0}\right|,\left|X u-p_{0}\right|\right) d \xi\right)^{\frac{1}{2}} \\
\leq & C(L, m) \sup _{B_{\rho}\left(\xi_{0}\right)}|X \varphi|\left|B_{\rho}\left(\xi_{0}\right)\right|_{G}\left(f_{B_{\rho}\left(\xi_{0}\right)}\left|V(X u)-V\left(p_{0}\right)\right|^{2} d \xi\right)^{\frac{1}{2}} \\
& \times\left(f_{B_{\rho}\left(\xi_{0}\right)} \omega\left(\left|p_{0}\right|,\left|X u-p_{0}\right|\right) d \xi\right)^{\frac{1}{2}} \\
\leq & C(L, m) \sup _{B_{\rho}\left(\xi_{0}\right)}|X \varphi|\left|B_{\rho}\left(\xi_{0}\right)\right|_{G} \Phi^{\frac{1}{2}}\left(\xi_{0}, \rho, p_{0}\right) \\
& \times \omega^{\frac{1}{2}}\left(\left|p_{0}\right|,\left(f_{B_{1}}\left|X u-p_{0}\right|^{2} d \xi\right)^{\frac{1}{2}}+\left(\int_{B_{2}}\left|X u-p_{0}\right|^{m} d \xi\right)^{\frac{1}{m}}\right) \\
\leq & C(L, m, M) \sup _{B_{\rho}\left(\xi_{0}\right)}|X \varphi|\left|B_{\rho}\left(\xi_{0}\right)\right|_{G} \Phi^{\frac{1}{2}}\left(\xi_{0}, \rho, p_{0}\right) \omega^{\frac{1}{2}}\left(\left|p_{0}\right|, \Phi^{\frac{1}{2}}\left(\xi_{0}, \rho, p_{0}\right)\right),
\end{aligned}
$$

where we have used estimates (2.10) and (2.11) in the last inequality.

By employing (H3), estimates (2.10) and (2.11), Young's inequality, and noting the fact that $K(\cdot)$ is monotone nondecreasing and $K(\cdot)>1$ and that $\rho \leq 1$, we deduce

$$
\begin{aligned}
I I^{\prime} \leq & \int_{B_{\rho}\left(\xi_{0}\right)} K(\cdot) \mu(\rho)\left(1+\left|p_{0}\right|\right)(1+|X u|)^{\frac{m}{2}} d \xi \\
\leq & K(\cdot) \mu(\rho)\left(1+\left|p_{0}\right|\right)^{1+\frac{m}{2}}\left|B_{\rho}\left(\xi_{0}\right)\right|_{G}+\int_{B_{1}+B_{2}} K(\cdot) \mu(\rho)\left(1+\left|p_{0}\right|\right)\left|X u-p_{0}\right|^{\frac{m}{2}} d \xi \\
\leq & K(\cdot) \mu(\rho)\left(1+\left|p_{0}\right|\right)^{1+\frac{m}{2}}\left|B_{\rho}\left(\xi_{0}\right)\right|_{G}+\left[K(\cdot) \mu(\rho)\left(1+\left|p_{0}\right|\right)\right]^{2}\left|B_{2}\right|_{G} \\
& +\left[K(\cdot) \mu(\rho)\left(1+\left|p_{0}\right|\right)\right]^{\frac{4}{4-m}}\left|B_{1}\right|_{G}+\int_{B_{2}}\left|X u-p_{0}\right|^{m} d \xi+\int_{B_{1}}\left|X u-p_{0}\right|^{2} d \xi \\
\leq & \left|B_{\rho}\left(\xi_{0}\right)\right|_{G} \Phi\left(\xi_{0}, \rho, p_{0}\right)+3\left[K(\cdot)\left(1+\left|p_{0}\right|\right)\right]^{2}\left|B_{\rho}\left(\xi_{0}\right)\right|_{G} \mu(\rho),
\end{aligned}
$$

where we have used $4 /(4-m)<2,1+m / 2<2$ and $\mu(\rho) \leq 1$ for $\rho \in[0,1]$.

Similarly to (3.23) to estimate $I I I^{\prime}$, the domain $B_{\rho}\left(\xi_{0}\right)$ is divided into four parts as previously mentioned. Then we obtain that, by Lemma 2(6) and Lemma 5 ,

$$
\begin{aligned}
I I I^{\prime} & \leq \sup _{B_{\rho}\left(\xi_{0}\right)}|X \varphi| \int_{B_{\rho}\left(\xi_{0}\right)} K(\cdot)(1+|X u|)^{m / 2} \mu(|v|) d \xi \\
& \leq \sup _{B_{\rho}\left(\xi_{0}\right)}|X \varphi| \int_{B_{\rho}\left(\xi_{0}\right)}\left[\frac{|v|}{\rho} \sqrt{\rho}+K^{2}(\cdot)\left(1+p_{0}\right)^{m} \mu(\sqrt{\rho})+K^{2}(\cdot)\left|X u-p_{0}\right|^{m} \mu(\sqrt{\rho})\right] d \xi
\end{aligned}
$$




$$
\begin{aligned}
\leq & \sup _{B_{\rho}\left(\xi_{0}\right)}|X \varphi|\left[C(m, M)\left(C_{P}+K^{2}(\cdot)\right) \int_{B_{\rho}\left(\xi_{0}\right)}\left|V\left(X u-p_{0}\right)\right|^{2} d \xi\right. \\
& \left.+K^{4 /(2-m)}(\cdot)\left(2+p_{0}\right)^{m} \mu(\sqrt{\rho})\left|B_{\rho}\left(\xi_{0}\right)\right|_{G}\right] \\
\leq & \sup _{B_{\rho}\left(\xi_{0}\right)}|X \varphi|\left[C(m, M)\left(C_{P}+K^{2}(\cdot)\right) \Phi\left(\xi_{0}, p_{0}, \rho\right)\left|B_{\rho}\left(\xi_{0}\right)\right|_{G}\right. \\
& \left.+K^{4 /(2-m)}(\cdot)\left(2+p_{0}\right)^{m} \mu(\sqrt{\rho})\left|B_{\rho}\left(\xi_{0}\right)\right|_{G}\right] .
\end{aligned}
$$

With the help of the assumptions that $\sup _{B_{\rho}\left(\xi_{0}\right)}|\varphi| \leq \rho \leq 1$, Hölder's inequality, Sobolev type inequality and Young's inequality, we get

$$
\begin{aligned}
I V^{\prime} \leq & C \int_{B_{\rho}\left(\xi_{0}\right)}\left(|X u|^{m\left(1-\frac{1}{r}\right)}+|u|^{r-1}+1\right)|\varphi| d \xi \\
\leq & C\left(\int_{B_{\rho}\left(\xi_{0}\right)}|X u|^{m} d \xi\right)^{\left(1-\frac{1}{r}\right)}\left(\int_{B_{\rho}\left(\xi_{0}\right)}|\varphi|^{r} d \xi\right)^{\frac{1}{r}}+C \rho\left|B_{\rho}\left(\xi_{0}\right)\right|_{G}\left[1+\left(\left|u_{0}\right|+\left|p_{0}\right|\right)^{r-1}\right] \\
& +C\left(\int_{B_{\rho}\left(\xi_{0}\right)}\left|u-u_{0}-p_{0}\left(\xi^{1}-\xi_{0}^{1}\right)\right|^{r} d \xi\right)^{\left(1-\frac{1}{r}\right)}\left(\int_{B_{\rho}\left(\xi_{0}\right)}|\varphi|^{r} d \xi\right)^{\frac{1}{r}} \\
\leq & C\left(\int_{B_{\rho}\left(\xi_{0}\right)}\left(\left|X u-p_{0}\right|^{m}+\left|p_{0}\right|^{m}\right) d \xi\right)^{\left(1-\frac{1}{r}\right)}\left(\int_{B_{\rho}\left(\xi_{0}\right)}|\varphi|^{r} d \xi\right)^{\frac{1}{r}} \\
& +C\left(\int_{B_{\rho}\left(\xi_{0}\right)}\left|X u-p_{0}\right|^{m} d \xi\right)^{\frac{r-1}{m}}\left(\int_{B_{\rho}\left(\xi_{0}\right)}|\varphi|^{r} d \xi\right)^{\frac{1}{r}} \\
& +C \rho\left|B_{\rho}\left(\xi_{0}\right)\right|_{G}\left[1+\left(\left|u_{0}\right|+\left|p_{0}\right|\right)^{r-1}\right] \\
\leq & C\left(\int_{B_{\rho}\left(\xi_{0}\right)}\left|X u-p_{0}\right|^{m} d \xi\right)^{\frac{r-1}{r}}\left(\int_{B_{\rho}\left(\xi_{0}\right)}|\varphi|^{r} d \xi\right)^{\frac{1}{r}} \\
& +C\left(\int_{B_{\rho}\left(\xi_{0}\right)}\left|p_{0}\right|^{m} d \xi\right)^{\frac{r-1}{r}}\left(\int_{B_{\rho}\left(\xi_{0}\right)}|\varphi|^{r} d \xi\right)^{\frac{1}{r}} \\
\leq & C \int_{B_{\rho}\left(\xi_{0}\right)}\left|X u-p_{0}\right|^{m} d \xi+C \rho^{r}\left|B_{\rho}\left(\xi_{0}\right)\right|_{G} \\
& +C \rho\left|B_{\rho}\left(\xi_{0}\right)\right|_{G}\left[1+\left(\left|u_{0}\right|+\left|p_{0}\right|\right)^{r-1}+\left|p_{0}\right|^{m\left(1-\frac{1}{r}\right)}\right] . \\
& +C\left(\int_{B_{\rho}\left(\xi_{0}\right)}\left|X u-p_{0}\right|^{m} d \xi\right)^{\frac{r-1}{m}}\left(\int_{B_{\rho}\left(\xi_{0}\right)}|\varphi|^{r} d \xi\right)^{\frac{1}{r}} \\
+ & C \rho\left|B_{\rho}\left(\xi_{0}\right)\right|_{G}\left[1+\left(\left|u_{0}\right|+\left|p_{0}\right|\right)^{r-1}\right] \\
& C\left(\int_{B_{\rho}\left(\xi_{0}\right)}\left|X u-p_{0}\right|^{m} d \xi\right)^{\frac{r-1}{r}}\left(\int_{B_{\rho}\left(\xi_{0}\right)}|\varphi|^{r} d \xi\right)^{\frac{1}{r}} \\
+ & C \rho\left|B_{\rho}\left(\xi_{0}\right)\right|_{G}\left[1+\left(\left|u_{0}\right|+\left|p_{0}\right|\right)^{r-1}+\left|p_{0}\right|^{m\left(1-\frac{1}{r}\right)}\right] \\
& \\
& \\
& \\
&
\end{aligned}
$$

On the case $B_{1}=: B_{\rho}\left(\xi_{0}\right) \cap\left\{\left|X u-p_{0}\right| \leq 1\right\}$, by (2.10) and Young's inequality, one gets

$$
\left|X u-p_{0}\right|^{m} \leq\left|X u-p_{0}\right|^{2}+1 \leq\left|V(X u)-V\left(p_{0}\right)\right|^{2}+1,
$$


and then

$$
\begin{aligned}
(4.9) \leq & C \mu(\sqrt{\rho})\left|B_{\rho}\left(\xi_{0}\right)\right|\left[\left(1+\left|u_{0}\right|+\left|p_{0}\right|\right)^{r-1}+\left(1+\left|u_{0}\right|+\left|p_{0}\right|\right)^{m}\right] \\
& +C\left|B_{\rho}\left(\xi_{0}\right)\right|_{G} \Phi\left(\xi_{0}, \rho, p_{0}\right) .
\end{aligned}
$$

On the other hand, on $B_{2}=: B_{\rho}\left(\xi_{0}\right) \cap\left\{\left|X u-p_{0}\right|>1\right\}$, by (2.11) and Young's inequality, one has

$$
\begin{aligned}
(4.9) \leq & C \mu(\sqrt{\rho})\left|B_{\rho}\left(\xi_{0}\right)\right|\left[\left(1+\left|u_{0}\right|+\left|p_{0}\right|\right)^{r-1}+\left(1+\left|u_{0}\right|+\left|p_{0}\right|\right)^{m}\right] \\
& +C\left|B_{\rho}\left(\xi_{0}\right)\right|_{G} \Phi\left(\xi_{0}, \rho, p_{0}\right) .
\end{aligned}
$$

Thus we infer that, by combining these estimates and noting the definition of $F(s, t)$, we have

$$
I V^{\prime} \leq C \Phi\left(\xi_{0}, \rho, p_{0}\right)\left|B_{\rho}\left(\xi_{0}\right)\right|_{G}+C F\left(\left|u_{0}\right|,\left|p_{0}\right|\right)\left|B_{\rho}\left(\xi_{0}\right)\right|_{G} \mu(\sqrt{\rho}) .
$$

Combining the estimates $I^{\prime}, I I^{\prime}, I I I^{\prime}$ and $I V^{\prime}$ with (4.3), we immediately conclude (4.1).

We next establish an initial excess improvement estimate assuming that the excess $\Phi$ is initially small enough. Precisely,

Lemma 9 (Excess improvement) Let $u \in H W^{1, m}\left(\Omega, \mathbb{R}^{N}\right)$ satisfy the conditions of Theorem 1 . Assume that Lemma 4 and the following smallness conditions hold:

$$
\begin{aligned}
& \omega^{\frac{1}{2}}\left(\left|u_{\xi_{0}, \rho}\right|+\left|(X u)_{\xi_{0}, \rho}\right|, \Phi^{\frac{1}{2}}\left(\xi_{0}, \rho,(X u)_{\xi_{0}, \rho}\right)\right)+\Phi^{1 / 2}\left(\xi_{0}, \rho,(X u)_{\xi_{0}, \rho}\right) \leq \frac{\delta}{2}, \\
& C_{2} F^{2}\left(\left|u_{\xi_{0}, \rho}\right|,\left|(X u)_{\xi_{0}, \rho}\right|\right) \mu(\sqrt{\rho}) \leq \delta^{2}
\end{aligned}
$$

with $C_{2}=8 C_{1}^{2} C_{4}$, together with the condition

$$
\rho \leq \rho_{1}^{\frac{m}{(2-m)(m-1)}}\left(1+\left|u_{\xi_{0}, \rho}\right|, 1+\left|(X u)_{\xi_{0}, \rho}\right|\right) \text {. }
$$

Then the following growth inequality is valid for $\tau \in[\gamma, 1)$ :

$$
\Phi\left(\xi_{0}, \theta \rho,(X u)_{\xi_{0}, \theta \rho}\right) \leq \theta^{2 \tau} \Phi(\rho)+K^{*}\left(\left|u_{\xi_{0}, \rho}\right|,\left|(X u)_{\xi_{0}, \rho}\right|\right) \mu^{2}\left(\rho^{\sigma}\right)
$$

where $\sigma=\min \{(2-m)(m-1) / m,(m-1) / 2\}$, and $K^{*}(s, t)=C_{8} F^{\frac{2 m}{m-1}}(1+s, M+t)$.

Proof For simplicity, we will use the abbreviation $\Phi(\rho)=\Phi\left(\xi_{0}, \rho,(X u)_{\xi_{0}, \rho}\right)$ in the sequel. For $\varepsilon>0$ to be determined later, we take $\delta \in(0,1)$ and $\Upsilon \in[0,1]$ to be the corresponding constant from the $\mathcal{A}$-harmonic approximation lemma (Lemma 4), and set

$$
w=u-\left(u_{\xi_{0}, \rho}-\Upsilon h_{\xi_{0}, 2 \theta \rho}\right)-(X u)_{\xi_{0, \rho}}\left(\xi^{1}-\xi_{0}^{1}\right)
$$

and

$$
\Upsilon=C(m, M) \Gamma(\rho), \quad \Gamma(\rho)=C_{1} \sqrt{\Phi(\rho)+4 \delta^{-2} \mu^{2}\left(\sqrt{\rho) F^{2}\left(\left|u_{\xi_{0}, \rho}\right|,\left|(X u)_{\xi_{0}, \rho}\right|\right)} .\right.}
$$


Noting (4.13) and (4.14) yields

$$
\omega^{1 / 2}\left(\left|(X u)_{\xi_{0}, \rho}\right|, \Phi^{1 / 2}(\rho)\right)+\Phi^{1 / 2}(\rho) \leq \frac{\delta}{2}
$$

and by (4.1), we derive (note the definition of $\Upsilon$ and $\Gamma(\rho)$ )

$$
\begin{aligned}
& \left|f_{B_{\rho}\left(\xi_{0}\right)}\left[A_{i, p_{\beta}^{j}}^{\alpha}\left(\xi_{0}, u_{\xi_{0}, \rho},(X u)_{\xi_{0}, \rho}\right) X w\right] X_{i} \varphi^{\alpha} d \xi\right| \\
& \quad \leq \Upsilon \frac{\omega^{1 / 2}\left(\left|(X u)_{\xi_{0}, \rho}\right|, \Phi^{1 / 2}(\rho)\right) \Phi^{1 / 2}(\rho)+\Phi(\rho)+\mu(\sqrt{\rho}) F\left(\left|u_{\xi_{0}, \rho}\right|,\left|(X u)_{\xi_{0}, \rho}\right|\right)}{C(m, M) \Gamma(\rho)} \sup _{B_{\rho}\left(\xi_{0}\right)}|X \varphi| \\
& \quad \leq \Upsilon\left[\omega^{1 / 2}\left(\left|(X u)_{\xi_{0}, \rho}\right|, \Phi^{1 / 2}(\rho)\right)+\Phi^{1 / 2}(\rho)+\frac{\delta}{2}\right] \sup _{B_{\rho}\left(\xi_{0}\right)}|X \varphi| \\
& \quad \leq \Upsilon \delta \sup _{B_{\rho}\left(\xi_{0}\right)}|X \varphi| .
\end{aligned}
$$

Then from the definition of $\Upsilon$, Lemma 2(6) and (2.9), we have

$$
f_{B_{\rho}\left(\xi_{0}\right)}|W(X w)|^{2} d \xi \leq f_{B_{\rho}\left(\xi_{0}\right)}|V(X w)|^{2} d \xi \leq C(m, M) \Phi(\rho) \leq \Upsilon^{2}
$$

Inequalities (4.18) and (4.19) fulfill the conditions of $\mathcal{A}$-harmonic approximation lemma, which ensures than we find an $\mathcal{A}=A_{i, p_{\beta}^{j}}^{\alpha}\left(\xi_{0}, u_{\xi_{0}, \rho},(X u)_{\xi_{0}, \rho}\right)$-harmonic function $h \in W^{1, m}\left(B_{\rho}\left(\xi_{0}\right), \mathbb{R}^{N}\right)$ such that

$$
f_{B_{\rho}\left(\xi_{0}\right)}|W(X h)|^{2} d \xi \leq 1, \quad f_{B_{\rho}\left(\xi_{0}\right)}\left|W\left(\frac{w-\Upsilon h}{\rho}\right)\right|^{2} d \xi \leq \Upsilon^{2} \varepsilon
$$

Using Lemma 2(3) and (6), we have

$$
\begin{aligned}
\Phi(\theta \rho)= & f_{B_{\theta \rho}\left(\xi_{0}\right)}\left|V(X u)-V\left((X u)_{\xi_{0, \theta \rho}}\right)\right|^{2} d \xi \\
\leq & C f_{B_{\theta \rho}\left(\xi_{0}\right)}\left|V\left(X u-(X u)_{\xi_{0, \theta \rho}}\right)\right|^{2} d \xi \\
\leq & C f_{B_{\theta \rho}\left(\xi_{0}\right)}\left|V\left(X u-(X u)_{\xi_{0, \rho}}-\Upsilon(X h)_{\left(\xi_{0}, 2 \theta \rho\right)}\right)\right|^{2} d \xi \\
& +C\left|V\left((X u)_{\xi_{0, \theta \rho}}-(X u)_{\xi_{0, \rho}}-\Upsilon(X h)_{\left(\xi_{0}, 2 \theta \rho\right)}\right)\right|^{2},
\end{aligned}
$$

where the constant $C$ depends only on $m, k$ and $N$.

Next, we proceed to estimate the right-hand side of (4.21). Decomposing $B_{\theta \rho}\left(\xi_{0}\right)$ into two parts: $B_{1}=B_{\theta \rho}\left(\xi_{0}\right) \cap\left\{\left|X u-(X u)_{\xi_{0, \rho}}-\Upsilon(X h)_{\left(\xi_{0}, 2 \theta \rho\right)}\right| \leq 1\right\}$ and $B_{2}=B_{\theta \rho}\left(\xi_{0}\right) \cap$ $\left\{\left|X u-(X u)_{\xi_{0, \rho}}-\Upsilon(X h)_{\left(\xi_{0}, 2 \theta \rho\right)}\right|>1\right\}$. Then by Lemma 2(1) and Hölder inequality, we obtain

$$
\begin{aligned}
& \left|(X u)_{\xi_{0, \theta} \rho}-(X u)_{\xi_{0, \rho}}-\Upsilon(X h)_{\left(\xi_{0}, 2 \theta \rho\right)}\right| \\
& \quad \leq f_{B_{\theta \rho}\left(\xi_{0}\right)}\left|X u-(X u)_{\xi_{0, \rho}}-\Upsilon(X h)_{\left(\xi_{0}, 2 \theta \rho\right)}\right| d \xi
\end{aligned}
$$




$$
\begin{aligned}
\leq & \sqrt{2} f_{B_{1}}\left|V\left(X u-(X u)_{\xi_{0, \rho}}-\Upsilon(X h)_{\left(\xi_{0}, 2 \theta \rho\right)}\right)\right| d \xi \\
& +\sqrt[m]{2} f_{B_{2}}\left|V\left(X u-(X u)_{\xi_{0, \rho}}-\Upsilon(X h)_{\left(\xi_{0}, 2 \theta \rho\right)}\right)\right|^{2 / m} d \xi \\
\leq & \sqrt[m]{2}\left(\Xi^{1 / 2}+\Xi^{1 / m}\right),
\end{aligned}
$$

where we have denoted

$$
\Xi=: f_{B_{\theta \rho}\left(\xi_{0}\right)}\left|V\left(X u-(X u)_{\xi_{0}, \rho}-\Upsilon(X h)_{\left(\xi_{0}, 2 \theta \rho\right)}\right)\right|^{2} d \xi
$$

Using Lemma 2(1), we deduce that $V^{2}\left(\Xi^{1 / 2}+\Xi^{1 / m}\right) \leq C\left(\Xi+\Xi^{2 / m}\right)$. So we get

$$
\Phi(\theta \rho) \leq C\left(\Xi+V^{2}\left(\Xi^{1 / 2}+\Xi^{1 / m}\right)\right) \leq C_{3}\left(\Xi+\Xi^{2 / m}\right)
$$

where the constant $C_{3}$ depends only on $m, Q$ and $N$. Then it remains for us to estimate $\Xi$. By considering the cases $|X h| \leq 1$ and $|X h|>1$, separately and keeping in mind (4.20), we obtain

$$
f_{B_{\rho}\left(\xi_{0}\right)}|X h| d \xi \leq 2 \sqrt{2} f_{B_{\rho}\left(\xi_{0}\right)}|W(X h)|^{2} d \xi \leq 2 \sqrt{2},
$$

where we have used Lemma 2(1).

Note that the smallness conditions (4.13) and (4.14) imply $C_{4} \Upsilon^{2} \leq 1$ with $C_{4}=$ $\max \left\{8 C_{0}^{2},(2 \theta)^{-Q}\right\}$, where we have assumed $\frac{1}{2} C_{1}^{2} C_{4} \delta^{2} \leq 1$, which is no restriction. Then it follows by applying the priori estimate for constant coefficients sub-elliptic systems (see Lemma 6)

$$
\Upsilon\left|(X h)_{\left(\xi_{0}, 2 \theta \rho\right)}\right| \leq \Upsilon \sup _{B_{\rho / 2}\left(\xi_{0}\right)}|X h| \leq \Upsilon C_{0} f_{B_{\rho}\left(\xi_{0}\right)}|X h| d \xi \leq 2 \sqrt{2} \Upsilon C_{0} \leq 1
$$

Caccioppoli type inequality applied on $B_{2 \theta \rho}\left(\xi_{0}\right)$ with $u_{0}=u_{\xi_{0}, \rho}$, and $p_{0}=(X u)_{\xi_{0}, \rho}+$ $\Upsilon(X h)_{\xi_{0}, 2 \theta \rho}, \theta \in(0,1 / 4]$ yields

$$
\begin{aligned}
\Xi \leq & C_{c}\left[\left|B_{2 \theta \rho}\left(\xi_{0}\right)\right|_{G}^{-1} \int_{B_{2 \theta \rho}\left(\xi_{0}\right)}\left|V\left(\frac{u-u_{\xi_{0}, \rho}-\left((X u)_{\xi_{0}, \rho}+\Upsilon(X h)_{\left(\xi_{0}, 2 \theta \rho\right)}\right)\left(\xi^{1}-\xi_{0}^{1}\right)}{2 \theta \rho}\right)\right|^{2} d \xi\right. \\
& +U]
\end{aligned}
$$

where

$$
\begin{aligned}
U= & {\left[K\left(\left|u_{\xi_{0}, \rho}\right|+\left|(X u)_{\xi_{0}, \rho}+\Upsilon(X h)_{\left(\xi_{0}, 2 \theta \rho\right)}\right|\right)\left(1+\left|(X u)_{\xi_{0}, \rho}+\Upsilon(X h)_{\left(\xi_{0}, 2 \theta \rho\right)}\right|\right)\right]^{2 m /(m-1)} } \\
& \times \mu^{2}\left((2 \theta \rho)^{(2-m)(m-1) / m}\right) \\
& +\left(1+2 M+\left|(X u)_{\xi_{0}, \rho}+\Upsilon(X h)_{\left(\xi_{0}, 2 \theta \rho\right)}\right|\right)^{m /(m-1)^{2}}(2 \theta \rho)^{m-1} \\
& +\mu^{2}\left((2 \theta \rho)^{(2-m)(m-1) / m}\right)\left[f_{B_{\rho}\left(\xi_{0}\right)}\left(|X u|^{m}+|u|^{r}+1\right) d \xi\right]^{\frac{m}{m-1}\left(1-\frac{1}{r}\right)} .
\end{aligned}
$$


By Lemma 2(3), one gets

$$
\begin{gathered}
\int_{B_{2 \theta \rho}\left(\xi_{0}\right)}\left|V\left(\frac{u-u_{\xi_{0}, \rho}-\left((X u)_{\xi_{0}, \rho}+\Upsilon(X h)_{\left(\xi_{0}, 2 \theta \rho\right)}\right)\left(\xi^{1}-\xi_{0}^{1}\right)}{2 \theta \rho}\right)\right|^{2} d \xi \\
\leq \int_{B_{2 \theta \rho}\left(\xi_{0}\right)}\left|V\left(\frac{u-\left(u_{\xi_{0}, \rho}-\Upsilon h_{\xi_{0}, 2 \theta \rho}\right)-(X u)_{\xi_{0}, \rho}\left(\xi^{1}-\xi_{0}^{1}\right)-\Upsilon h(\xi)}{2 \theta \rho}\right)\right|^{2} d \xi \\
\left.+\frac{\Upsilon h(\xi)-\Upsilon h_{\xi_{0}, 2 \theta \rho}-\Upsilon(X h)_{\left(\xi_{0}, 2 \theta \rho\right)}\left(\xi^{1}-\xi_{0}^{1}\right)}{2 \theta \rho}\right) \\
\leq C\left[\int _ { B _ { 2 \theta \rho } ( \xi _ { 0 } ) } \left(\left|V\left(\frac{w-\Upsilon h(\xi)}{2 \theta \rho}\right)\right|^{2}\right.\right. \\
\left.\left.+\left|V\left(\Upsilon \frac{h(\xi)-h_{\xi_{0}, 2 \theta \rho}-(X h)_{\left(\xi_{0}, 2 \theta \rho\right)}\left(\xi^{1}-\xi_{0}^{1}\right)}{2 \theta \rho}\right)\right|^{2}\right) d \xi\right] .
\end{gathered}
$$

To estimate the right-hand side, we employ (2.9), Lemma 2(2) (note that $\frac{1}{2 \theta} \geq 1$ ) and (4.20) to infer

$$
\begin{aligned}
f_{B_{2 \theta \rho}\left(\xi_{0}\right)}\left|V\left(\frac{w-\Upsilon h(\xi)}{2 \theta \rho}\right)\right|^{2} d \xi & \leq C(2 \theta)^{-Q-2} f_{B_{\rho}\left(\xi_{0}\right)}\left|W\left(\frac{w-\Upsilon h(\xi)}{\rho}\right)\right|^{2} d \xi \\
& \leq C(2 \theta)^{-Q-2} \Upsilon^{2} \varepsilon .
\end{aligned}
$$

Using Lemma 2, Sobolev-Poincare type inequality (3.6), Lemma 6, (2.9) and (4.20) leads to

$$
\begin{aligned}
& f_{B_{2 \theta \rho}\left(\xi_{0}\right)}\left|V\left(\Upsilon \frac{h(\xi)-h_{\xi_{0}, 2 \theta \rho}-(X h)_{\left(\xi_{0}, 2 \theta \rho\right)}\left(\xi^{1}-\xi_{0}^{1}\right)}{2 \theta \rho}\right)\right|^{2} d \xi \\
& \quad \leq C_{P}^{2} \Upsilon^{2} f_{B_{2 \theta \rho}\left(\xi_{0}\right)}\left|V\left(X h(\xi)-(X h)_{\left(\xi_{0}, 2 \theta \rho\right)}\right)\right|^{2} d \xi \\
& \leq C_{P}^{4}(2 \theta \rho)^{2} \Upsilon^{2} f_{B_{2 \theta \rho}\left(\xi_{0}\right)}\left|V\left(X^{2} h\right)\right|^{2} d \xi \\
& \leq C_{P}^{4}(2 \theta \rho)^{2} \Upsilon^{2} \sup _{B_{\rho / 2}\left(\xi_{0}\right)}\left|X^{2} h\right|^{2} \\
& \leq C_{0} C_{P}^{4}(2 \theta)^{2} \Upsilon^{2} f_{B_{\rho}\left(\xi_{0}\right)}|(X h)|^{2} d \xi \\
& \leq C C_{0} C_{P}^{4}(2 \theta)^{2} \Upsilon^{2} f_{B_{\rho}\left(\xi_{0}\right)}|W(X h)|^{2} d \xi \\
& \leq C_{5} \theta^{2} \Upsilon^{2},
\end{aligned}
$$

where we denote $C_{5}=4 C C_{0} C_{P}^{4}$.

Using (4.25), we get

$$
\begin{aligned}
{[K(\mid} & \left.\left.u_{\xi_{0}, \rho}|+|(X u)_{\xi_{0}, \rho}+\Upsilon(X h)_{\left(\xi_{0}, 2 \theta \rho\right)} \mid\right)\left(1+\left|(X u)_{\xi_{0}, \rho}+\Upsilon(X h)_{\left(\xi_{0}, 2 \theta \rho\right)}\right|\right)\right]^{2 m /(m-1)} \\
& \times \mu^{2}\left((2 \theta \rho)^{(2-m)(m-1) / m}\right) \\
\leq & {\left[K\left(\left|u_{\xi_{0}, \rho}\right|+\left|(X u)_{\xi_{0}, \rho}\right|+1\right)\left(2+\left|(X u)_{\xi_{0}, \rho}\right|\right)\right]^{2 m /(m-1)} \mu^{2}\left(\rho^{(2-m)(m-1) / m}\right) } \\
\leq & F^{2 m /(m-1)}\left(1+\left|u_{\xi_{0}, \rho}\right|,\left|(X u)_{\xi_{0}, \rho}\right|\right) \mu^{2}\left(\rho^{(2-m)(m-1) / m}\right)
\end{aligned}
$$


and

$$
\begin{aligned}
(1 & \left.+2 M+\left|(X u)_{\xi_{0}, \rho}+\Upsilon(X h)_{\left(\xi_{0}, 2 \theta \rho\right)}\right|\right)^{m /(m-1)^{2}}(2 \theta \rho)^{m-1} \\
& \leq\left(2+2 M+\left|(X u)_{\xi_{0}, \rho}\right|\right)^{m /(m-1)^{2}} \mu^{2}\left(\rho^{(m-1) / 2}\right) .
\end{aligned}
$$

Applying Sobolev type inequality, we have

$$
\begin{aligned}
& {\left[f_{B_{2 \theta \rho}\left(\xi_{0}\right)}\left(|X u|^{m}+|u|^{r}+1\right) d \xi\right]^{m(r-1) / r(m-1)} } \\
& \leq {\left[2^{m-1} f_{B_{2 \theta \rho}\left(\xi_{0}\right)}\left(\left|X u-(X u)_{\xi_{0}, \rho}\right|^{m}\right) d \xi\right]^{m(r-1) / r(m-1)} } \\
&+\left(2^{m-1}\left|(X u)_{\xi_{0}, \rho}\right|^{m}\right)^{m(r-1) / r(m-1)} \\
&+\left[f_{B_{2 \theta \rho}\left(\xi_{0}\right)} 2^{r-1}\left(\left|u-u_{\xi_{0}, \rho}-(X u)_{\xi_{0}, \rho}\left(\xi^{1}-\xi_{0}^{1}\right)\right|^{r}\right) d \xi\right]^{m(r-1) / r(m-1)} \\
&+\left(2^{r-1}\left|u_{\xi_{0}, \rho}+(X u)_{\xi_{0}, \rho}\left(\xi^{1}-\xi_{0}^{1}\right)\right|^{r}+1\right)^{m(r-1) / r(m-1)} \\
& \leq\left(2^{m-1}\right)^{m(r-1) / r(m-1)}\left[(2 \theta)^{-Q} f_{B_{\rho}\left(\xi_{0}\right)}\left|X u-(X u)_{\xi_{0}, \rho}\right|^{m} d \xi\right]^{m(r-1) / r(m-1)} \\
&+\left(2^{m-1}\left|(X u)_{\xi_{0}, \rho}\right|^{m}\right)^{m(r-1) / r(m-1)} \\
&+\left(2^{r-1}\right)^{m(r-1) / r(m-1)}\left[(2 \theta)^{-Q} f_{B_{\rho}\left(\xi_{0}\right)}\left|X u-(X u)_{\xi_{0}, \rho}\right|^{m} d \xi\right]^{(r-1) /(m-1)} \\
&+\left(2^{r-1}\left|u_{\xi_{0}, \rho}+(X u)_{\xi_{0}, \rho}\right|^{r}+1\right)^{m(r-1) / r(m-1)} \\
& \leq C(m, M, Q)\left[(2 \theta)^{-Q} \Phi\left(\xi_{0}, \rho,(X u)_{\xi_{0}, \rho}\right)\right]^{m(r-1) / r(m-1)} \\
&+C(m, Q)\left(1+\left|u_{\xi_{0}, \rho}\right|+\left|(X u)_{\xi_{0}, \rho}\right|\right)^{m(r-1) /(m-1)} .
\end{aligned}
$$

The smallness conditions imply

$$
(2 \theta)^{-Q} \Phi\left(\xi_{0}, \rho,(X u)_{\xi_{0}, \rho}\right) \leq 1
$$

And then it follows

$$
\begin{aligned}
& {\left[f_{B_{2 \theta_{\rho}\left(\xi_{0}\right)}}\left(|X u|^{m}+|u|^{r}+1\right) d \xi\right]^{m(r-1) / r(m-1)} \mu^{2}\left((2 \theta \rho)^{(2-m)(m-1) / m}\right)} \\
& \quad \leq C F^{\frac{m}{m-1}}\left(\left|u_{\xi_{0}, \rho}\right|,\left|(X u)_{\xi_{0}, \rho}\right|\right) \mu^{2}\left(\rho^{(2-m)(m-1) / m}\right),
\end{aligned}
$$

where we have used the definition of $F$ in the first step.

Combining all the above estimates with (4.26) and letting $\varepsilon=\theta^{Q+4}$, we arrive at

$$
\Xi \leq C_{6}\left[\theta^{2} \Upsilon^{2}+F^{\frac{2 m}{m-1}}\left(1+\left|u_{\xi_{0}, \rho}\right|, M+\left|(X u)_{\xi_{0}, \rho}\right|\right) \mu^{2}\left(\rho^{\sigma}\right)\right]
$$

where $\sigma=\min \{(2-m)(m-1) / m,(m-1) / 2\}, C_{6}$ depends only on $Q, N, m, M, L, \lambda$ and $C_{P}$. For given $\tau \in[\gamma, 1)$, choosing $\theta \in\left(0, \frac{1}{4}\right)$ suitable such that $C_{3} C_{6} \theta^{2} \leq \theta^{2 \tau}$, we easily find 
(note the definition of $\Upsilon$ )

$$
\begin{aligned}
\Phi(\theta \rho) & \leq \theta^{2 \tau}\left[\Phi(\rho)+C_{7} F^{\frac{2 m}{m-1}}\left(1+\left|u_{\xi_{0}, \rho}\right|, M+\left|(X u)_{\xi_{0}, \rho}\right|\right) \mu^{2}\left(\rho^{\sigma}\right)\right] \\
& :=\theta^{2 \tau} \Phi(\rho)+K^{*}\left(\left|u_{\xi_{0}, \rho}\right|,\left|(X u)_{\xi_{0}, \rho}\right|\right) \mu^{2}\left(\rho^{\sigma}\right),
\end{aligned}
$$

where we have used that $(2-m)(m-1) / m \leq 3-2 \sqrt{2}<1 / 2, K^{*}(s, t)=C_{7} F^{\frac{2 m}{m-1}}(1+s, M+t)$, and the constant $C_{7}$ has the same dependencies as $C_{6}$.

For $T>0$, we find $\Phi_{0}(T)>0$ (depending on $Q, N, \lambda, L, \tau$ and $\omega$ ) such that

$$
\begin{aligned}
& \omega^{\frac{1}{2}}\left(2 T, 2 \Phi_{0}^{\frac{1}{2}}(T)\right)+2 \Phi_{0}^{\frac{1}{2}}(T) \leq \frac{1}{2} \delta, \quad \text { and } \\
& 2\left(1+\sqrt{C_{p}}\right) \sqrt{\Phi_{0}(T)} \leq \theta^{Q / 2}\left(1-\theta^{\tau}\right) T .
\end{aligned}
$$

With $\Phi_{0}(T)$ from (4.33) and (4.34), we choose $\rho_{0}(T) \in(0,1]$ (depending on $Q, N, \lambda, L, \tau$, $\omega, \eta$ and $\kappa)$ such that

$$
\begin{aligned}
& \rho_{0}(T) \leq \rho_{1}^{(2-m)(m-1) / m}(1+2 T, 1+2 T), \\
& C_{2} F^{2}(2 T, 2 T) \mu^{2}\left(\rho_{0}(T)\right) \leq \delta^{2}, \\
& K_{0}(T) \mu^{2}\left(\rho_{0}(T)^{\sigma}\right) \leq\left(\theta^{2 \gamma}-\theta^{2 \tau}\right) \Phi_{0}(T), \quad \text { and } \\
& 2\left(1+C_{p}\right) K_{0}(T) H\left(\rho_{0}(T)^{2}\right) \leq \theta^{Q}\left(1-\theta^{\gamma}\right)^{2}\left(\theta^{2 \gamma}-\theta^{2 \tau}\right) T^{2},
\end{aligned}
$$

where $K_{0}(T):=K^{*}(2 T, 2 T)$.

The rest of the process to obtain Theorem 1 is very similar to [13]. We omit it here.

\section{Conclusion}

For ELLIPTIC systems in Euclidean spaces, there are many literature sources to study partial regularity of weak solutions by the so-called $\mathcal{A}$-harmonic approximation method. With respect to SUB-ELLIPTIC equations and systems in Heisenberg groups, or general Carnot groups, there are some new difficulties and challenges which remain due to the lack of commutation and homogeneity of the horizontal vector fields. This work is concerned with nonlinear sub-elliptic systems (1.1) with Dini continuous coefficients which are weaker than Hölder continuous coefficients. Under sub-quadratic controllable growth conditions, a $C^{1}$ partial regularity result is obtained by adapting the technique of $\mathcal{A}$-harmonic approximation to our setting. The new result generalizes the corresponding result for elliptic systems in Euclidean spaces and enriches regularity theory for nonlinear sub-elliptic systems in Carnot groups. The authors also believe that this work helps further study nonlinear sub-elliptic systems structured on Hörmander vector fields. 


\section{Abbreviations}

Not applicable.

\section{Availability of data and materials}

Not applicable.

\section{Competing interests}

The authors declare that there are no competing interests regarding the publication of this article.

\section{Authors' contributions}

The work presented here was carried out in collaboration among all authors. JL found the motivation of this paper and suggested the outline of the proofs. DN provided good ideas for completing this paper. YQ and SM gave some valuable suggestions for completing the paper. All authors have contributed to, read and approved the final version.

\section{Author details}

'School of Mathematics and Computer Science, Gannan Normal University, Ganzhou, Jiangxi 341000, P.R. China. ${ }^{2}$ School of Physics and Electronic Information Technology, Gannan Normal University, Ganzhou, Jiangxi 341000, P.R. China.

\section{Publisher's Note}

Springer Nature remains neutral with regard to jurisdictional claims in published maps and institutional affiliations.

Received: 12 August 2017 Accepted: 5 October 2017 Published online: 16 October 2017

\section{References}

1. Wang, J, Niu, P: Optimal partial regularity for weak solutions of nonlinear sub-elliptic systems in Carnot groups. Nonlinear Anal. TMA 72, 4162-4187 (2010)

2. Wang, J, Liao, D, Yu, Z: Hölder continuity for sub-elliptic systems under the sub-quadratic controllable growth in Carnot groups. Rend. Semin. Mat. Univ. Padova 130, 169-202 (2013)

3. De Giorgi, E: Un esempio di estremali discontinue per un problem variazionale di upo ellitico. Boll. Unione Mat. Ital. 4, 135-137 (1968)

4. Giaquinta, M: Multiple Integrals in the Calculus of Variations and Nonlinear Elliptic Systems. Princeton University Press, Princeton (1983)

5. Giaquinta, M: Introduction to Regularity Theory for Nonlinear Elliptic Systems. Birkhäuser, Berlin (1993)

6. Chen, Y, Wu, L: Second Order Elliptic Equations and Elliptic Systems. Science Press, Beijing (2003)

7. Duzaar, F, Steffen, K: Optimal interior and boundary regularity for almost minimizers to elliptic variational integrals. J. Reine Angew. Math. 546, 73-138 (2002)

8. Duzaar, F, Grotowski, JF: Partial regularity for nonlinear elliptic systems: the method of A-harmonic approximation. Manuscr. Math. 103, 267-298 (2000)

9. Duzaar, F, Grotowski, JF, Kronz, M: Regularity of almost minimizers of quasi-convex variational integrals with subquadratic growth. Ann. Mat. Pura Appl. 184, 421-448 (2005)

10. Duzaar, F, Mingione, G: The $p$-harmonic approximation and the regularity of $p$-harmonic maps. Calc. Var. Partial Differ. Equ. 20, 235-256 (2004)

11. Duzaar, F, Mingione, G: Regularity for degenerate elliptic problems via $p$-harmonic approximation. Ann. Inst. Henri Poincaré, Anal. Non Linéaire 21, 735-766 (2004)

12. Chen, $\mathrm{S}$, Tan, Z: The method of A-harmonic approximation and optimal interior partial regularity for nonlinear elliptic systems under the controllable growth condition. J. Math. Anal. Appl. 335, 20-42 (2007)

13. Duzaar, F, Gastel, A: Nonlinear elliptic systems with Dini continuous coefficients. Arch. Math. 78, 58-73 (2002)

14. Duzaar, F, Gastel, A, Mingione, G: Elliptic systems, singular sets and Dini continuity. Commun. Partial Differ. Equ. 29 1215-1240 (2004)

15. Qiu, Y: Optimal partial regularity of second order nonlinear elliptic systems with Dini continuous coefficients for the superquadratic case. Nonlinear Anal. TMA 75, 3574-3590 (2012)

16. Capogna, L, Garofalo, N: Regularity of minimizers of the calculus of variations in Carnot groups via hypoellipticity of systems of Hörmander type. J. Eur. Math. Soc. 5, 1-40 (2003)

17. Shores, E: Regularity theory for weak solutions of systems in Carnot groups. PhD thesis, University of Arkansas (2005)

18. Föglein, A: Partial regularity results for subelliptic systems in the Heisenberg group. Calc. Var. Partial Differ. Equ. 32 , 25-51 (2008)

19. Zheng, S, Feng, Z: Regularity of subelliptic $p$-harmonic systems with subcritical growth in Carnot group. J. Differ. Equ. $258,2471-2494(2015)$

20. Domokos, A: On the regularity of $p$-harmonic functions in the Heisenberg group. PhD thesis, University of Pittsburgh (2004)

21. Capogna, L: Regularity for quasilinear equation and 1-quasiconformal maps in Carnot groups. Math. Ann. 313 263-295 (1999)

22. Manfredi, J, Mingione, G: Regularity results for quasilinear elliptic equations in the Heisenberg group. Math. Ann. 339, 485-544 (2007)

23. Mingione, G, Zatorska-Goldstein, A, Zhong, X: Gradient regularity for elliptic equations in the Heisenberg group. Adv. Math. 222, 62-129 (2009)

24. Di Fazio, G, Fanciullo, MS: Cordes nonlinear operators in Carnot groups. Electron. J. Differ. Equ. 2015, 191 (2015)

25. $\mathrm{Yu}, \mathrm{H}$, Zheng, S: Morrey estimates for subelliptic $p$-Laplace type systems with VMO coefficients in Carnot groups. Electron. J. Differ. Equ. 2016, 33 (2016)

26. Bal, K: Uniqueness of a positive solution for quasilinear elliptic equations in Heisenberg group. Electron. J. Differ. Equ. 2016, $130(2016)$ 
27. Ferrara, M, Bisci, GM, Repovs, D: Nonlinear elliptic equations on Carnot groups. Rev. R. Acad. Cienc. Exactas Fís. Nat., Ser. A Mat. 111, 707-718 (2017)

28. Katzourakis, N: The sub-elliptic $\infty$-Laplace system on Carnot-Carathéodory spaces. Adv. Nonlinear Anal. 2, 213-233 (2013)

29. Bisci, GM, Repovs, D: Yamabe-type equations on Carnot groups. Potential Anal. 46, 369-383 (2017)

30. Tyagi, J: Nontrivial solutions for singular semilinear elliptic equations on the Heisenberg group. Adv. Nonlinear Anal. 2 , 87-94 (2014)

31. Wang, J, Liao, D: Optimal partial regularity for nonlinear sub-elliptic systems with Dini continuous coefficients in Carnot groups. Bound. Value Probl. 2016, 18 (2016). doi:10.1186/s13661-016-0525-7

32. Folland, G: Subelliptic estimates and function spaces on nilpotent Lie groups. Ark. Mat. 13, 161-207 (1975)

33. Carozza, M, Fusco, N, Mingione, G: Partial regularity of minimizers of quasiconvex integrals with subquadratic growth. Ann. Mat. Pura Appl. 175, 141-164 (1998)

34. Acerbi, E, Fusco, N: Regularity for minimizers of nonquadratic functionals: the case $1<p<2$. J. Math. Anal. Appl. 140, 115-135 (1989)

\section{Submit your manuscript to a SpringerOpen ${ }^{\circ}$ journal and benefit from:}

- Convenient online submission

$\checkmark$ Rigorous peer review

- Open access: articles freely available online

- High visibility within the field

- Retaining the copyright to your article

Submit your next manuscript at $\gg$ springeropen.com 